\title{
Are losartan and Imatinib Effective Against SARS-CoV2 Pathogenesis? A Pathophysiologic-Based In Silico Study
}

Reza Nejat ${ }^{1^{*}}$, Ahmad Shahir Sadr $2,3,4,5^{*}$

${ }^{1}$ Anesthesiologist, Fellow of Critical Care Medicine, Former Assistant Professor,Department of Anesthesiology and Critical Care Medicine, Shahid Beheshti University of Medical Sciences, Tehran, Iran.

${ }^{2}$ Bioinformatics Research Center, Cheragh Medical Institute and Hospital, Kabul, Afghanistan.

${ }^{3}$ Department of Computer Science, Faculty of Mathematical Sciences, Shahid Beheshti University, Tehran, Iran.

${ }^{4}$ Department of Phytochemistry, Medicinal Plants and Drugs Research Institute, Shahid Beheshti University, Tehran, Iran.

${ }^{5}$ School of Biological Sciences, Institute for Research in Fundamental Sciences (IPM), Tehran, Iran.

*. indicate equal senior authors.

\begin{abstract}
AIM: Proposing a theory about the pathophysiology of cytokine storm in COVID19, we were to find the potential drugs to treat this disease and to find any effect of these drugs on the virus infectivity through an In Silico study.

Subject and Method: COVID-19-induced ARDS is linked to a cytokine storm phenomenon not explainable solely by the virus infectivity. Knowing that ACE2, the hydrolyzing enzyme of Angll and SARS-CoV2 receptor, downregulates when the virus enters the host cells, we hypothesize that hyperacute Angll upregulation is the eliciting factor of this ARDS. We were to validate this theory through reviewing previous studies to figure out the role of overzealous activation of AT1R in ARDS. Imatinib, a tyrosine kinase inhibitor, has previously been elucidated to be promising in modulating lung inflammatory reactions and virus infectivity in SARS and MERS. We did an In Silico study to uncover any probable other unconsidered inhibitory effects of losartan and imatinib against SARS-CoV2 pathogenesis.
\end{abstract}

Results: Reviewing the literature, we could find that over-activation of AT1R could explain precisely the mechanism of cytokine storm in COVID19. Our In Silico study revealed that losartan and imatinib could probably:

1- decline SARS-CoV2 affinity to ACE2

2- inhibit the main protease and furin,

3- disturb papain-like protease and p38MAPK functions, 
Conclusions: Our reviewing on renin-angiotensin system showed that overzealous activation of AT1R by hyper-acute excess of Angll due to acute downregulation of ACE2 by SARS-CoV2 explains precisely the mechanism of cytokine storm in COVID-19. Besides, based on our In Silico study we concluded that losartan and imatinib are promising in COVID19.

\section{Introduction}

Since December 2019, a viral disease called COVID19 has hunted thousands of people savagely all around the world and the death toll increases in a skyrocketing manner every day. It was elucidated that a coronavirus named SARS-CoV2 caused the disease eventually named as COVID-19. ${ }^{[1,2]}$ Coronaviruses are classified in the order Nidovirales, the suborder Coronavirinae, Coronaviridae family, the subfamily Orthocoronavirinae with four genera: Alphacoronavirus $(\alpha \mathrm{CoV})$, Betacoronavirus $(\beta \mathrm{CoV})$, Deltacoronavirus $(\delta \mathrm{CoV})$, and Gammacoronavirus ( $\mathrm{YCoV})$. SARS-CoV2, along with SARS-CoV and MERS-CoV, belongs to $\beta$-genus. ${ }^{[1,3]}$

These pathogens are large, enveloped and positive-sense single stranded RNA viruses. $[1,4]$ The genome of SARS-CoV2 consists of 29891 nucleotides encoding 9860 amino acids with genomic sequence of:[1]

5'-replicase (orf1/ab)-structural proteins [Spike (S)-Envelope (E)- Membrane [2]-Nucleocapsid (N)]-3'

Two main open-reading frames (ORFs), ORF1a and ORF1b of the nucleotide sequence the virus genome are translated into two co-terminal polyproteins ppl1a (ORF1a) and ppl1ab (ORF1a \& ORF1b together). These polyproteins are cleaved into non-strucrtural proteins (NSPs) by two proteases: a papain-like protease (PLpro) and the main serine type protease (Mpro). ${ }^{[1],[5-7]}$

Intriguingly, PLpro and some nsps (nsp3) apart from their crucial role in replication of the virus $[1,5,8]$, provide the virus with deubiquitinating and delSGylating properties. ${ }^{[9,10]}$ These viral proteins inhibit Toll-like receptor3 (TLR3) and Toll-like receptor7 (TLR7), as well. ${ }^{[5,6]}$ In vivo, it has been demonstrated that type I and type III interferon responses against SARS-CoV2 is lower than that of seen in respiratory syncytial virus (RSV) and influenza A virus. It seems that SARS-CoV2 like SARS-CoV evade innate immunity successfully. ${ }^{[11,12]}$

Viral genomic and subgenomic RNA synthesis leads to production of structural proteins, membrane $(M)$, nucleocapsid $(N)$, envelope $(E)^{[7]}$ structural proteins which determine the different compartments and shape of the virus. Furthermore, a glycoprotein structure called spike (S) protein protruding from the surface of the virus determines the host range, tissue tropism and the host immune responses. ${ }^{[13]}$ As a class 1 fusion protein, $S$ protein of coronaviruses is composed of two trimeric subunits, $S_{1}$ and $S_{2}$. $S_{1}$, containing the receptor binding domain (RBD), mediates the attachment of the virus to its receptor on the host cell. $S_{2}$, the stalk of $S$ glycoprotein, is responsible for virus-cell fusion. ${ }^{[14-16]}$ As a mandatory step in pathogenesis of SARS-CoV2, cleavage of $S$ protein into $S_{1}$ and $S_{2}$ subunits as well as in $S_{2}$, immediately upstream to fusion peptide in $S_{2}$, plays an essential part in entry of the virus to the host cell and in cell-cell fusion and transmission. Furin 
(found abundantly in the lungs) and transmembrane serine protease-2 (TMPRSS2) contribute to this cleaving process crucially. ${ }^{[17-19]}$

The receptor of SARS-CoV2 on the host cells, like SARS-CoV, is angiotensin converting enzyme 2 (ACE2), a member of renin-angiotensin system (RAS). ${ }^{[20-22]}$ RAS is a very complex network of systemic as well as local ligands and receptors. ${ }^{[23]}$ It contributes to the regulation of immune system and cytokine production, cardiovascular system, metabolism, cell growth, salt and electrolyte homeostasis and vascular resistance. ${ }^{[24-26]}$

ACE2 as a mono-carboxypeptidase which removes single amino acids from peptides of RAS is not inhibited by ACE inhibitors like captopril or lisinopril.[27-29] This mono-peptidase converts angiotensin I [1-10] and angiotensin II [1-8] to angiotensin [1-9] and angiotensin [1-7], respectively. ${ }^{[29,30]}$ ACE2 is a functional competitor of ACE as the former reduces available angiotensin I [1-10], the substrate of ACE, by converting it to a less active metabolite, angiotensin [1-9]. Opposing to ACE2, ACE degrades angiotensin [1-7] to inactive products like angiotensin [1-5]. ${ }^{[31,32]}$ Angiotensin [1-7] is considered as an active peptide in RAS with antioxidative, anti-inflammatory, antiproliferative/antifibrotic, potent vasodilatory, and anti-thrombotic properties which exerts most of its effect via Mas receptor. ${ }^{[32-35]}$ On the other hand, ACE increases Ang II with its oxidative, proinflammatory, proliferative/fibrotic, vasoconstrictive and thrombotic effects which are mostly exerted through activation of angiotensin II type 1 receptor (AT1R). ${ }^{[36-38]}$ Another receptor for Ang II called angiotensin II type 2 receptor (AT2R) with cell protective and some opposing post-receptor effects to AT1R is distributed in a limited number of organs. ${ }^{[39]}$ It is implicated that ACE2/Ang[1-7]/Mas axis plays a counter-regulatory role against ACE/Ang II[1-8]/AT1R signaling pathway. ${ }^{[28,40-42]}$

Attachment of protein S with ACE2 results in the downregulation of the latter. ${ }^{[43,44]}$ Internalization of this receptor with the virus into the host cell or upregulation of constitutive physiologic shedding of ACE2 in the airways are potential causes of this phenomenon. ${ }^{[43]}$ Downregulation of ACE2 deregulates the balance in local RAS pathways in favor of ACE/Ang II [1-8]/AT1R in the lungs. In this context, hyperacute upregulation of local intracrine Ang II [1-8]/AT1R in the setting of invasion of huge number of SARS-CoV2 is not encountered with appropriate negative physiological feedback with ACE2. Furthermore, Angll has been demonstrated to decline ACE2 expression and function via lysosomal degradation mediated by AT1R. ${ }^{[45]}$ Henceforth, overzealous stimulation of AT1R sets on fire locally to provoke lung inflammation through pro-inflammatory, cytokine inducing, proliferative, thrombotic and tissue destructive effects as well as activating platelet derived growth factor receptor (PDGFR). ${ }^{[46-48]}$

It is implied that SARS-CoV2:

1- evades innate immunity via avoiding IRF3, TLR3 and TLR7 pathways by its nsp3 and papain-like proteases and elicit a moderate immune response,

2- downregulates its receptor (ACE2) after attachment, thereby, dysregulates the balance between two opposing axes of local RAS in the lungs in favor of Angll with all of its destructive and fibrotic properties in the tissues. 
According to the fact that the virus does not elicit an initial innate immunity response to explain the eruption of cytokine storm and simultaneously deregulates RAS in favor of ACE/Angll[1-8]/AT1R axis, we hypothesized that overzealous increase in Ang II/angiotensin[1-7] ratio may erupt the cytokine storm. Therefore, blocking of AT1R with an angiotensin receptor blocker like losartan would suppress the post-receptor deleterious effects of Angll in favor of angiotensin[1-7] or activation of AT2R with tissue protective effects. Moreover, subsiding immunopathological changes by an immunomodulator such as imatinib which had been used previously in SARS and MERS might alleviate the severity of the disease and may reduce the morbidity and mortality rates in COVID 19. We also did In Silico studies on different stages of SARS-CoV2 replication cycle to see if there are any other pharmacodynamic properties of these drugs that might be promising in reducing the infectivity of this virus.

\section{Method:}

Proposing a new insight to SARS-CoV2 biology and the pathophysiology of cytokine storm and ARDS in COVID-19 to reach into a way to reduce the mortality and morbidity of this disease, we searched for review and original articles focused and on COVID-19, SARS, MERS, RAS, the effect of AT1R and Ang II in ARDS and inflammatory reactions as a target in search engines science direct, scopus database and google scholar from 1990 till now. After reaching into the conclusion that Ang II/AT1R pathway may explain cytokine storm and ARDS in COVID-19 and accordingly losartan and imatinib could attenuate ARDS in this disease, we did In Silico study to investigate whether losartan and imatinib have any probable unconsidered inhibiting effect against the replication of the virus.

\section{In Silico study}

\section{Preparation of the Protein Structures}

The required protein structures were obtained from Protein Data Bank ${ }^{[49]}$ (PDB) according to Table 1:

Table 1. The protein crystal structures used in this study

\begin{tabular}{|c|c|c|c|}
\hline Macromolecule & Sequence Length & Organism & ID \\
\hline $\begin{array}{l}\text { SARS-CoV-2 spike receptor-RBD bound to } \\
\text { ACE2 }\end{array}$ & $603 / 229$ & $\begin{array}{l}\text { Homosapiens/SARS- } \\
\text { Cov2 }\end{array}$ & $6 \mathrm{moj}[50]$ \\
\hline Angiotensin Converting Enzyme 2 (ACE2) & 615 & Homo sapiens & $\left.1 \mathrm{r} 4\right|^{[51]}$ \\
\hline COVID-19 main protease & 306 & SARS-Cov2 & $6 l u 7[52]$ \\
\hline MAP Kinase p38 & 379 & Homo sapiens & $1 a 9 u^{[53]}$ \\
\hline Furin & 482 & Homo sapiens & $6 h z b{ }^{[54]}$ \\
\hline Papain-like protease & 316 & SARS-CoV & $3 m j 5^{[55]}$ \\
\hline $\begin{array}{l}\text { Angiotensin II type } 1 \text { receptor \& } \\
\text { Angiotensin II }\end{array}$ & $425 / 8$ & Homo sapiens & $60 s 0[56]$ \\
\hline
\end{tabular}


Proteins were studied for the date of publishing, crystallography techniques, the resolution, accompaniment of predefined inhibitor in crystal ("Pre-inh") and any required reconstruction due to probable missing of amino acids in their sequence vs the sequence of reference protein. The structures were observed by visualizing softwares (UCSF chimera ${ }^{[57]}$, Pymol[58], Swiss-PdbViewer ${ }^{[59]}$ to determine their unique protein chains and whether the structure is accompanied by other undesired molecules like (water, ions....) and to purify selectively to achieve the most desirable structure.

The mutated new amino acids in the sequence of SARS-CoV2 RBD were replaced on SARS-CoV RBD; MD-simulation was performed for new structure

In the beginning of our study (mid of February 2020) due to the lack of crystasl structure of SARS-CoV2 RBD with complete amino acid sequence, we replaced 22 defined mutated amino acids in SARS-CoV2 RBD on the corresponding place on SARS-CoV RBD to achieve a RBD structure with the most similarity to the real crystal structure of SARS-CoV2 RBD, assuming that $S$ proteins in these two viruses are $76 \%$ homologous. MD simulation of $100 \mathrm{~ns}$ was performed after accomplishing this replacement to achieve a persistently stable structure with the most homology to RBD-ACE2 complex of SARSCoV2. RMSD, RMSF, $\mathrm{H}$-bonding and radius of gyration diagrams are available. Due to the publishing of crystal structure of RBD-ACE2 complex of SARS-CoV2 by X-ray diffraction (resolution of $2.45 \AA$ ) we quitted using the achieved RBD-ACE2 complex and continued our bioinformatic study on the new published one (Figure $S 3$ )(Figure $S 3$ ).

\section{Preparation of Losartan and Imatinib}

The drug structures were obtained from Structure Data Bank such as Pubchem database ${ }^{[60]}$ and Drug bank database(Table S 1 ) (Table S1). ${ }^{[61]}$ The structures of the drugs were imported through gauss view, and then fully optimized geometries and properties of the electronic and structural properties of two molecules were derived by means of the density functional theory (DFT) method ${ }^{[62]}$ with B3LYP ${ }^{[63]}$ and STO-3G basis sets. ${ }^{[64]}$ The calculations were carried out using the Gaussian 03 package ${ }^{[65]}$. The program Open Babe [66] was used to generate SMILES strings from the optimized structure to be used for a similarity study by drug bank Chemical Structure Search with 0.5 to 0.7 Similarity threshold.

\section{Molecular Docking Simulation}

Docking study was performed by AutoDock $4^{[67]}$ to find the suitable orientation of the molecules in the active site of the protein structures. AutoDockTools 1.5.4 (ADT) was used to prepare input PDBQT files and to calculate a grid box. A special grid map appropriate for each structural size (table 4) around the active site of proteins was defined. The center of the grid boxes was aligned to the coordinates of the "Pre-inh"' for each of the protein structures. For the case of ACE2 three grid boxes were defined in three 
dimensions as appeared in table 4. The first ACE2 grid box was set around the "Pre-inh" position. The position of losartan and imatinib with the most affinity for ACE2 in grid box 1 vs the position of predefined inhibitor was determined.The second and the third ACE2 grid boxes encompassed RBD binding site around $\alpha$-helix and the whole ACE2 molecule, respectively.

As PLpro inhibitory active site changed in 100ns MD simulation two grid boxes were defined based on the position of active site; one before MD simulation and the second after 100ns MD simulation. Binding energy for losartan and imatinib attachment with PLpro was calculated before and after MD simulation.

A Lamarckian genetic algorithm (LGA) was used for the searching of the status of binding sites. Every LGA job comprised of 250 runs. The final structures were grouped and classified according to the most favorable binding energy. This procedure was applied to the two drugs in a similar manner. A more negative score determines which of these drugs are more likely to dock with a protein structure (target protein) with subsequent more favorable interactions. The docking model of each protein complex with its "Pre-inh" was generated by AutoDock4 (Table 2). Reliability of the applied docking protocol was assessed by separation of "Pre-inh" from its protein structure and then redocking of each "Pre-inh" into the active site of the latter.

Table 2 grid box dimensions for each structure

\begin{tabular}{|l|l|c|c|c|}
\hline Macrmolcules & \multicolumn{1}{|c|}{ Steps } & Grid points & Spacing $\AA$ & Grid Center \\
\hline ACE2 & $1^{\text {st }}$ Docking & $80 \times 100 \times 80$ & 0.375 & $40 \times 6.0 \times 29$ \\
\hline ACE2 & $2^{\text {nd }}$ Docking & $75 \times 70 \times 80$ & 0.375 & $13 \times 15 \times 20$ \\
\hline ACE2 & $3^{\text {rd }}$ Docking & $120 \times 85 \times 100$ & 0.600 & $39 \times 3.0 \times 22$ \\
\hline Mpro & & $80 \times 100 \times 100$ & 0.375 & $-15 \times 13 \times 70$ \\
\hline p38MAPK & & $70 \times 70 \times 70$ & 0.375 & $4 \times 16 \times 29$ \\
\hline PLpro & Before MD & $80 \times 80 \times 80$ & 0.375 & $-13 \times 45 \times-36$ \\
\hline PLpro & After MD & $80 \times 80 \times 80$ & 0.375 & $40 \times 45 \times 47$ \\
\hline Furin & & $80 \times 100 \times 80$ & 0.375 & $40 \times 45 \times 47$ \\
\hline
\end{tabular}

After exposing of the two drugs with the protein structures, we obtained docking energy for each [drug structure] complex. The clusters of docking energies were determined for 250 posing status and the relevant numerical tables for each complex was studied.

\section{Simulation}

Molecular dynamic (MD) simulations, to study the dynamicity of the protein structures over a defined time period to characterize the behavior and stability of the structures was executed by Gromacs package for 100 nano-second (ns) in this study. 
MD simulations of the protein-drug complexes following docking were performed with the GROMACS 2018 package using the GROMOS96 43a1 force field. ${ }^{[68]}$ The conformation status for ACE2 and PLpro complexes with their ligands with the highest affinity were selected as the initial conformation for MD simulations. First the topology parameters of protein were created and the complex was immersed in a cubic box of simple point charge (SPC) water molecules. ${ }^{[69]}$ The "solvated system" (protein, ions, small molecule and water) was neutralized by adding required counter-ions $\mathrm{Na}$ or $\mathrm{Cl}$. To equilibrate the system, the solutes (Proteins, counterions, and two drugs) were subjected to the positionrestrained dynamics simulation (NVT and NPT) at $299.177 \mathrm{~K}$ for $1 \mathrm{~ns}$. Finally, the full system was subjected to an MD run for $100 \mathrm{~ns}$ at $300 \mathrm{~K}$ temperature and 1 bar pressure.

MD simulation was performed for ACE2 crystal, ACE2-SARS-CoV mutated RBD (refer to results), imatinib-ACE2, losartan-ACE2 and imatinib-PLpro, losartan-PLpro complexes.

\section{New ACE2 after MD simulation of ACE2-Iosartan and ACE2-imatinib complexes}

In order to study the persistency of losartan-ACE2 and imatinib-ACE2 complexes, we performed 100ns MD simulation for each complex. Two new ACE2 (nACE2) under the influence of each of these ligands were exported: losartan (Lo,nACE2) and imatinib (Im,nACE2). RMSD, RMSF, radius of gyration and h-bonding diagrams of each complex were obtained (Figure S 2)(Figure 2).

\section{Super-imposition of ACE2 Structures Before and After 100ns MD with Losartan and Imatinib}

After MD simulation of ACE2-losartan and ACE2-imatinib complexes, ACE2 was separated from the drugs. The structure of ACE2 under the influence of losartan and imatinib were superimposed on the structure of ACE2 crystal prior to any docking to evaluate the degree of changes in conformational shape of ACE2 in each complex.

\section{Losartan against other ARBs in docking with ACE2}

ARBs were searched for on Kyoto Encyclopedia of Genes and Genomes. ${ }^{70]}$ Docking was done for each member of ARBs with ACE2 crystal structure and the relevant energy binding for each item was obtained.

\section{Redocking study after MD simulation}

After MD simulation of complexes for 100ns, the ligands were separated. Through redocking, the binding energies and the binding status of the two drugs with the protein structures was evaluated. ${ }^{[71]}$

\section{Analysis}

RMSD, RMSF, hydrogen bonding and radius of gyration diagrams exported after performing MD simulation were analyzed by qtgrace. ${ }^{[72]}$ Ligplot $^{[73]}$ and poseview ${ }^{[74]}$ were used for determining the hydrogen bonding, hydrophobic and pi-pi interactions after docking and MD simulation. Visual analyzing was done with ucsf chimera and pymol. 
Exposure of imatinib and losartan with Mpro, furin, p38MAPK and PLpro

We exposed imatinib and losartan to crystal structure of Mpro, furin, p38MAPK and PLpro to figure out if the drugs would pose the site of their relevant "Pre-inh". It is worth mentioning that redocking of predefined inhibitor of furin in its crystal structure obtained from PDB was disturbed and showed error in the process. We optimized the structure and performed docking for the second round to earn a reliable reference for binding energy.

\section{MD simulation of imatinib and losartan with PLpro.}

We performed MD simulation for 100 ns after exposure of imatinib and losartan with PLpro. RMSD, RMSF and radius of gyration were obtained.

\section{Configurations of computational systems:}

In this study we used multiple computational systems by different configurations (Table $S$ 2(Table S2).

\section{Results:}

\section{Losartan and imatinib bind to ACE2 with low energy (high affinity) compared to} “Pre-inh".

Docking of losartan and imatinib molecules with ACE2 before MD simulations, showed that the binding energy in three grid boxes as appeared in (Table 3) for the two drugs is lower than that of "Pre-inh". It is implied that losartan and imatinib attach to ACE2 with higher affinity (Figure S 1 ) in comparison with "Pre-inh". It merits mentioning that affinity of losartan and imatinib around $\alpha$-helix of ACE2 is lower than the other two grid boxes.

Table 3. Docking Energies of Imatinib and Losartan with ACE2 in 3 distinct grid boxes

\begin{tabular}{|l|c|c|c|}
\hline $\begin{array}{l}\text { Small } \\
\text { Molecules }\end{array}$ & $\begin{array}{c}\text { Grid } \\
\text { Box1 }\end{array}$ & $\begin{array}{c}\text { Grid } \\
\text { Box2 }\end{array}$ & $\begin{array}{c}\text { Grid } \\
\text { Box3 }\end{array}$ \\
\hline Imatinib & -15.21 & -11.79 & -12.64 \\
\hline Losartan & -9.67 & -8.44 & -9.6 \\
\hline Pre-inh & -7.28 & & \\
\hline
\end{tabular}

Losartan and imatinib could change the conformational structure of ACE2 persistently.

The data showed that the conformational structure of ACE2 after binding with both losartan and imatinib changed significantly at the binding site of ACE2 and RBD (Figure 1). It is expected that these two drugs lengthen the binding distance between $\alpha$-helix of ACE2 and SARS-CoV2 RBD due to relocation of this part of ACE2. (Table S 3)(Table S3). 


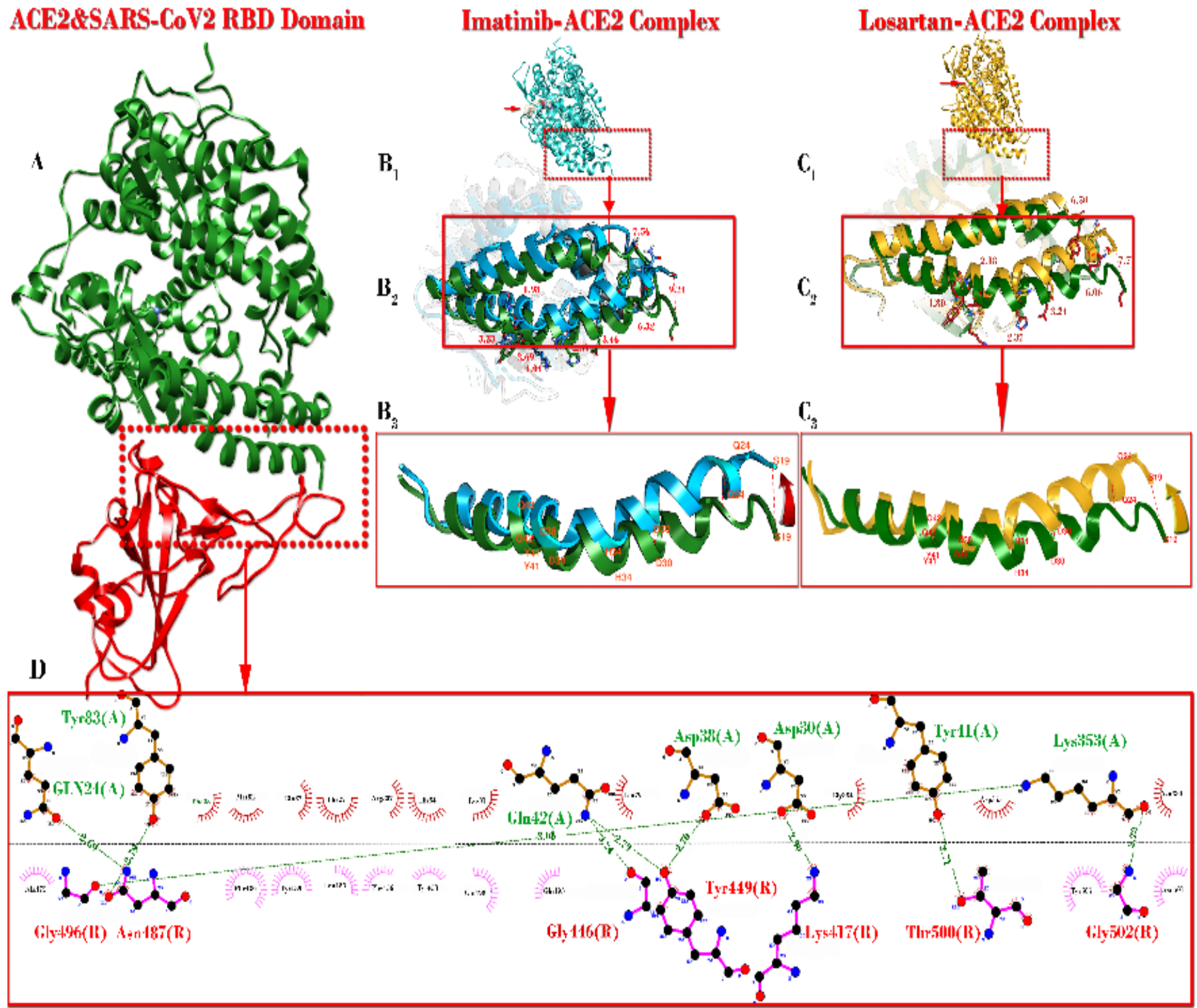

Figure 1. A:SARS-CoV2-ACE2 complex. B:1-Im,nACE2, 2-super-imposition of ACE2 and Im,nACE2, 3super-imposition of $\alpha$-helix of ACE2 and Im,nACE2. C:1-Lo,nACE2, 2-super-imposition of ACE2 and Lo.nACE2, 3-super-imposition of $\alpha$-helix of ACE2 and Lo,nACE2. D: hydrophobic and hydrogen bonds of SARS-CoV2RBD-ACE2

The binding energy of losartan and imatinib with Lo,nACE2 and Im,nACE2 after performing 100ns MD simulation changed.

The binding energies among losartan and imatinib with Lo,nACE2 and Im,nACE2 after redocking of losartan with Lo,nACE2, imatinib with Im,nACE2, losartan with Im,nACE2 and imatinib with Lo,nACE2 showed that the affinity of both drugs have increased with Lo,nACE2 and Im,nACE2 .(Table 4) (Table 4). 


\begin{tabular}{|l|l|l|}
\hline & \multicolumn{2}{|c|}{ Binding energy (Kcal/mole) } \\
\hline nACE2 & Losartan & Imatinib \\
\hline Lo,nACE2 & -11.99 & -14.25 \\
\hline Im,nACE2 & -8.47 & -17.78 \\
\hline
\end{tabular}

Available ARBs vs losartan could only bind to ACE2 with reasonable but lower affinity

Losartan in association with two unavailable ARBs, pratosartan and tasosartan, was in the upper three ranking of binding energy. Other ARBs bind with ACE2 with lower, yet reasonable energy ${ }^{[61]}$ (Table S 4) (Table S 4).

Imatinib and losartan could occupy the space where the "Pre-inh" in crystal structure of the main protease (Mpro) of SARS-CoV2 poses;

It was elucidated that imatinib as well as losartan had higher affinity to Mpro. Considering CADD theories, it shows that these ligands based on their affinity probably behave as inhibitors of Mpro, (Figure 2) 


\section{Docking of Mpro vs Imatinib and Losartan}
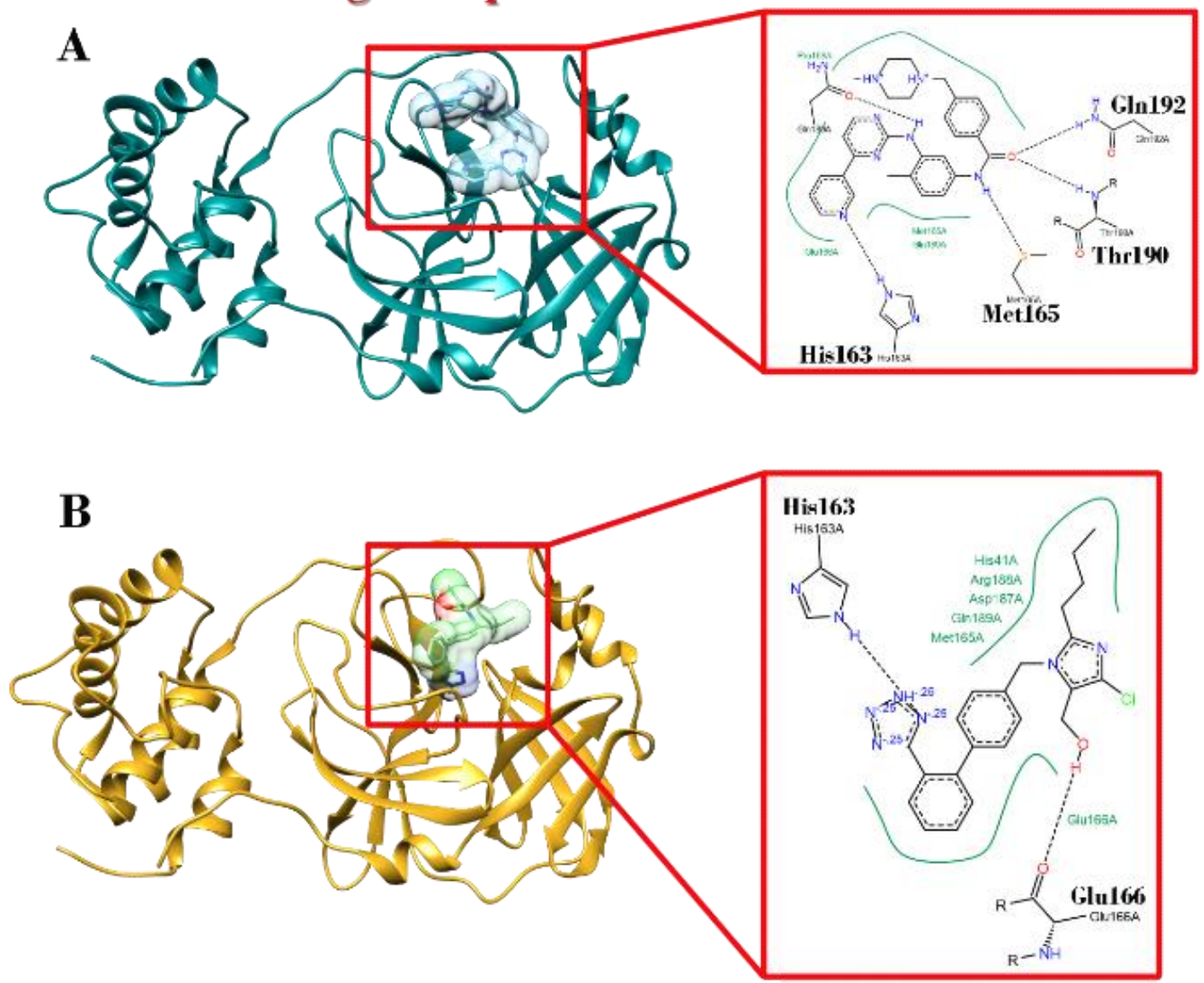

Figure 2. Main Protease (Mpro) of SARS-CoV2 complex with imatinib and Losartan.A:Ribbon view of complex and Poseview of interaction with imatinib B: Ribbon view of complex and Poseview of interaction with Losartan

Imatinib and losartan could occupy the space where the "Pre-inh" in crystal structure of the furin of SARS-CoV2 poses.

Our data demonstrated that imatinib had higher affinity for furin. But losartan showed up with lower but reasonable affinity. Considering CADD theories, it shows that imatinib based on its affinity will probably inhibit furin function. (Figure 3)(Table 5) 


\section{Docking of Furin by Imatinib \& Losartan}

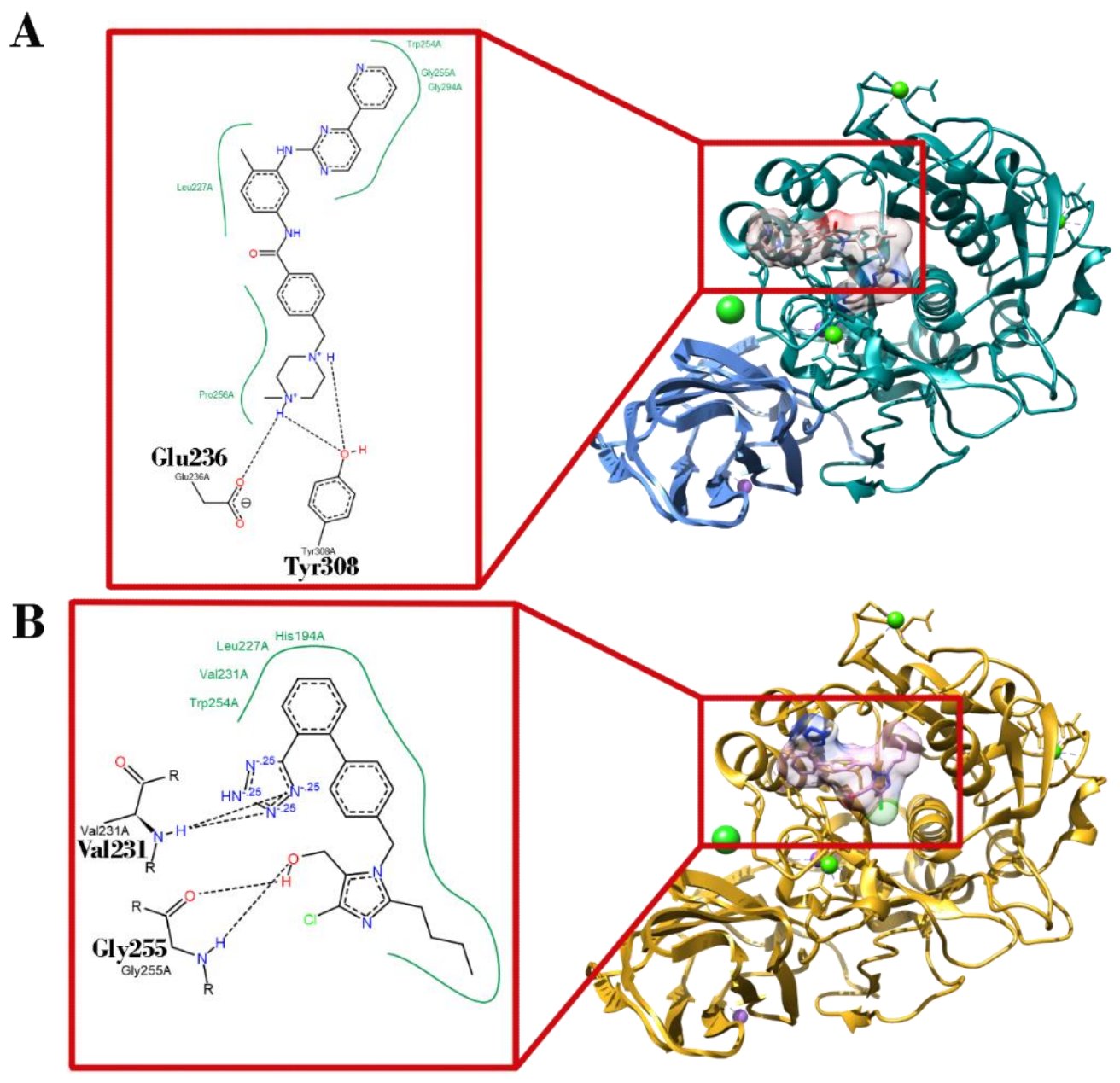

Figure 3. Position of Imatinib and Losartan in complex with furin with PDBID: 6hzb after Docking simulation. A:Ribbon view of complex and Poseview of interaction with imatinib B: Ribbon view of complex and Poseview of interaction with Losartan

Imatinib and losartan could occupy with higher affinity the space where the "Preinh" in crystal structure of p38MAPK poses

It was implicated that imatinib and losartan had higher affinity to p38 MAPK. Considering CADD theories, it shows that imatinib and losartan based on their affinity will probably inhibit p38MAPK function. (Figure 4)(Table 5) 


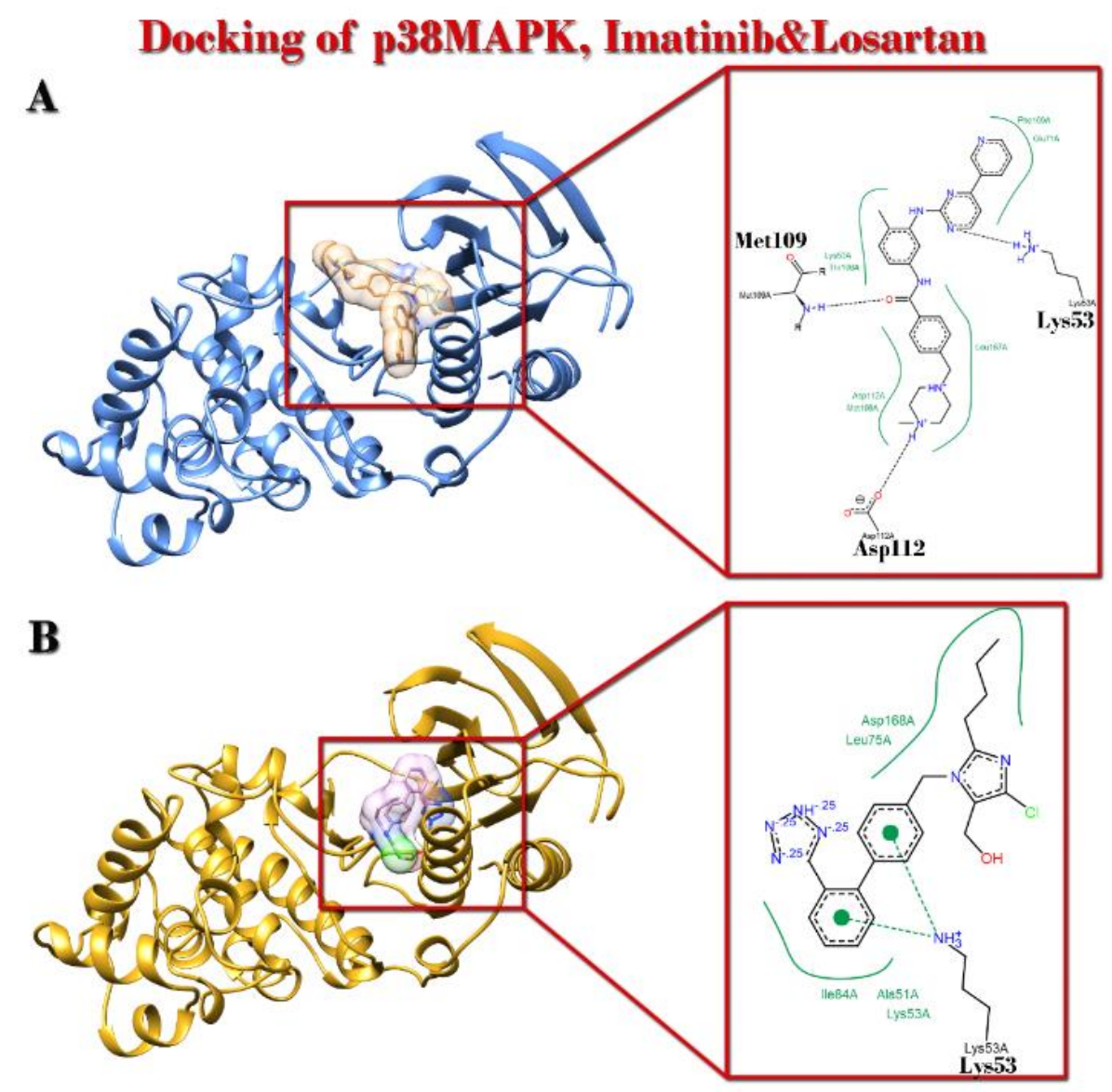

Figure 4. Position of Imatinib and Losartan in complex with p38MAPK with PDBID: 1a9u after Docking simulation. A:Ribbon view of complex and Poseview of interaction with imatinib B: Ribbon view of complex and Poseview of interaction with Losartan

Imatinib could occupy with higher affinity the space where "Pre-inh" in crystal structure of papin-like protease (PLpro) poses;

Imatinib showed higher affinity and losartan has lower affinity for PLpro relative to its "Preinh". Considering CADD theories imatinib might act as an inhibitor of PLpro function. (Table 5)(Figure 5,Figure 6)

\section{Losartan could change the conformational shape of PLpro}

After 100ns MD simulation exposure of losartan and PLpro, it was demonstrated that although affinity of losartan for PLpro is lower than that of imatinib and its "Pre-inh", this $A R B$ in 60 ns changes the radius of gyration and RMSD plots for at least $2.3 \mathrm{~A}^{\circ}$. It seems that losartan can disrupt PLpro. 


\section{Docking of PLpro, Imatinib \& Losartan}

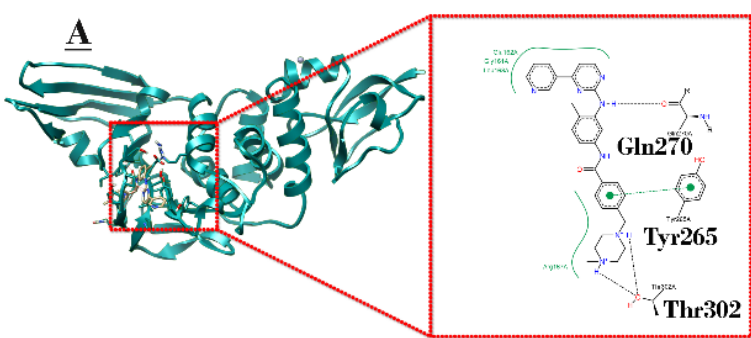

Before 100ns

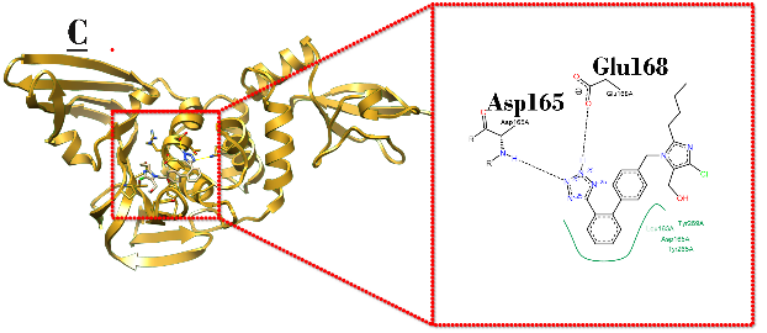

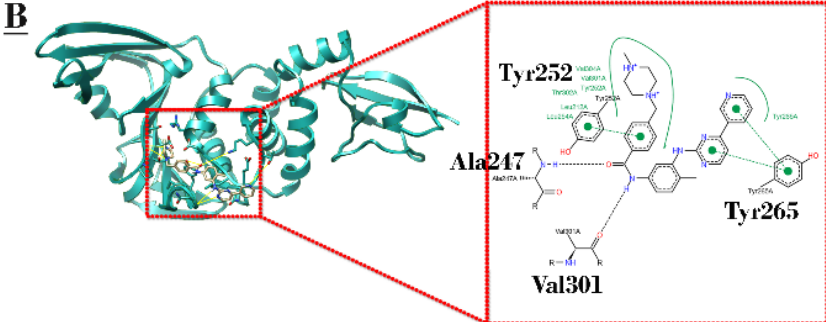

After 100ns

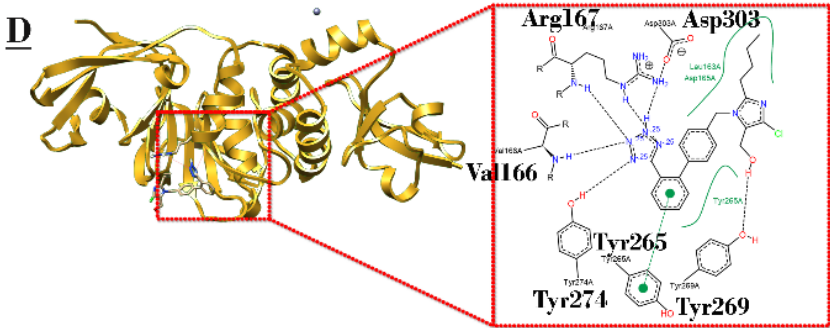

Figure 5. Position of Imatinib and Losartan in complex with PLpro with PDBID: $3 m j 5$, before and after 100ns MD simulation and redocking. A: Ribbon and poseview of complex of PLpro with imatinib before 100ns MD simulation, B: Ribbon and poseview of complex PLpro with imatinib after 100ns MD simulation C: Ribbon and poseview of complex of PLpro with losartan before 100ns MD simulation, D: Ribbon and poseview of complex PLpro with losartan after 100ns MD simulation

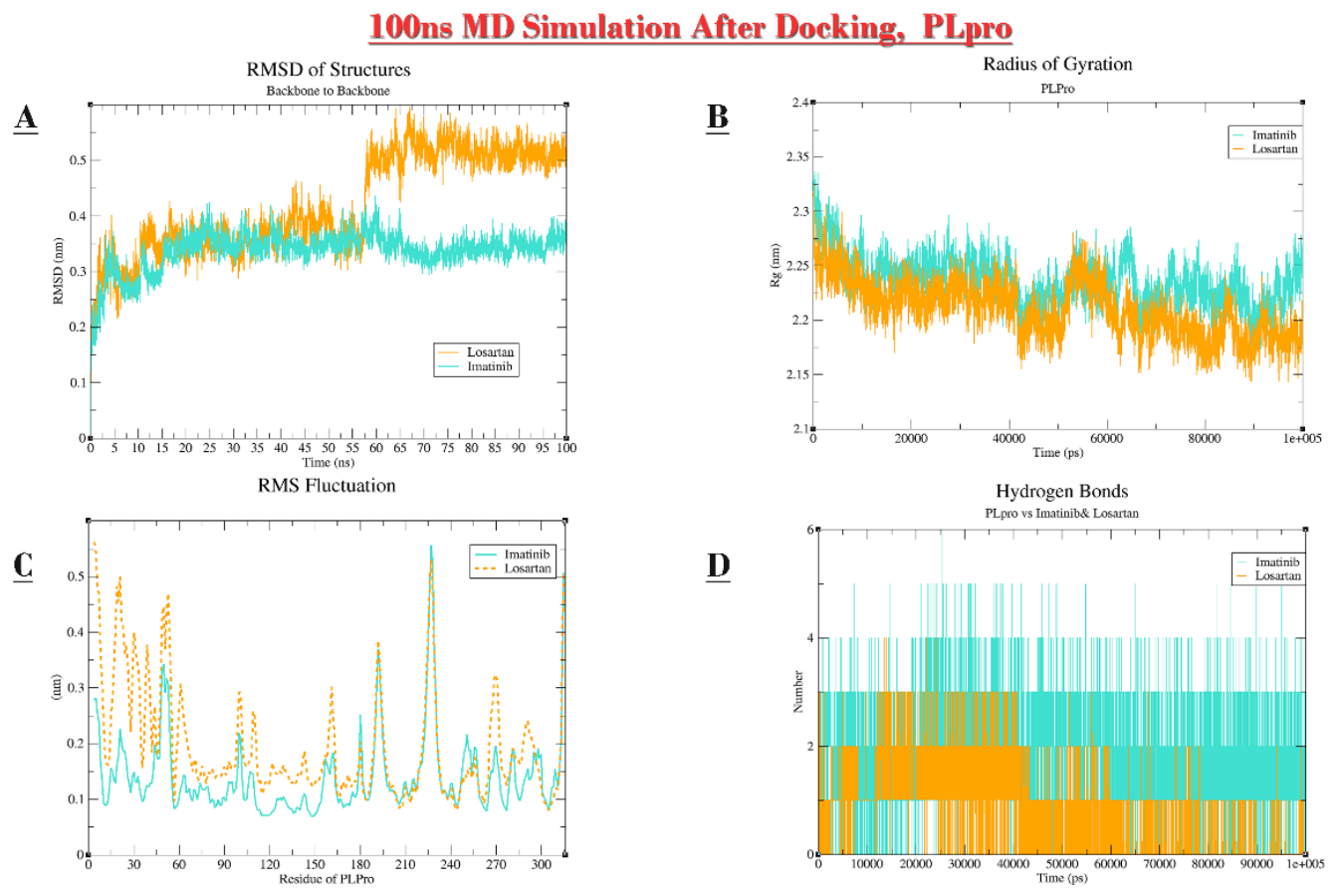

Figure 6. Diagrams of Losartan-PLpro and Imatinib-PLpro complexes after 100ns MD simulation;A: RMSD; B: Rg; C:RMSF; D: H-Bonding 


\section{DOCKING ENERGIES OF SMALL MOLECULES FOR BINDING WITH ALL TARGETS}

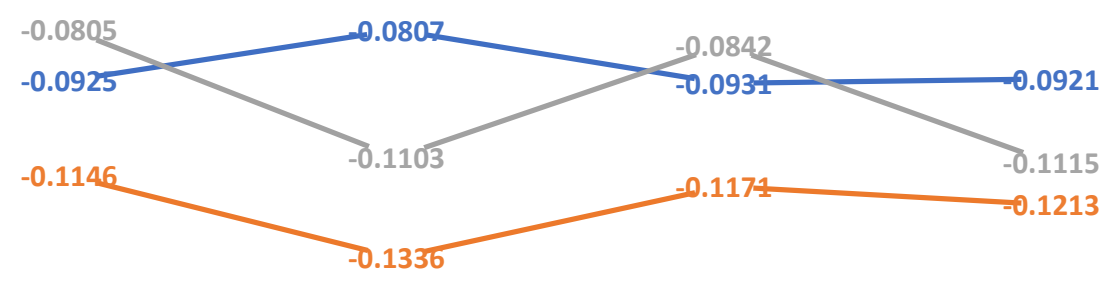

\begin{tabular}{|l|c|c|c|c|}
\cline { 2 - 5 } \multicolumn{1}{c|}{} & Mpro & Furin & p38MAPK & PLpro \\
\hline- - Losartan & -9.25 & -8.07 & -9.31 & -9.21 \\
\hline- - Imatinib & -11.46 & -13.36 & -11.71 & -12.13 \\
\hline- - Pre-Inh & -8.05 & -11.03 & -8.42 & -11.15 \\
\hline
\end{tabular}

\section{Angiotensin II (Ang II) - Imatinib Interaction}

We exposed imatinib and Ang II. The result shows that imatinib binds with Ang II with binding energy of $-8.64 \mathrm{Kcal} / \mathrm{mol}$ (Figure S 4 )(Figure S 4).

\section{Discussion}

\section{A new insight to pathophysiology of ARDS in COVID19}

The fatality and the change of the life style imposed by COVID19 have resulted in uprising death and economic burden in all countries. Despite the efforts done, the development of acute respiratory distress syndrome as the culprit of high morbidity and mortality in infection with SARS-CoV2 has remained elusive, yet attributed to a kind of cytokine storm. Apart from ARDS and cytokine storm, COVID19 seems to be a moderate illness, since SARS-CoV2 itself elicits a limited antiviral response in the hosts. ${ }^{[11]}$

Incubation of the virus in the upper respiratory tract results in the release of replicas into the lower airways and alveoli in huge number (about 10-1000 virion/ $\mu$ in its peak on day 5-6 of infection). ${ }^{[75]}$ ACE2, the virus receptor on host cells, besides to its expression on cardiovascular system, kidney, small intestine and adrenal, is also distributed in the apical portion of the ciliated nasal and tracheobronchial cells as well as pneumocytes type I and type II. ${ }^{[43,76,77]}$ Consequently, these immune competent cells of respiratory tract may harbor the virions easily. SARS-CoV2 entry to the cells is associated with downregulation of ACE2. This results in imbalance of ACE2/angiotensin[1-7]/Mas and ACE/Ang II/AT1R pathways in favor of the latter with pro-inflammatory, proliferative, prothrombotic, tissue destructive and pro-apoptotic properties. ${ }^{[44], ~[78] ~ I n ~ t h i s ~ c o n t e x t, ~ h y p e r a c u t e ~ e x c e s s ~ o f ~ A n g ~}$ 
Il seems to be the agent which can activate innate arm inflammatory cascades chaotically which results in sequential cytokine secretion. This process, as is not triggered directly by the antigens in a pathogen and originates from a collateral imbalance of hormonal milieu with immunological consequences, eventuates to the cytokine storm if not halted by negative feedback from activation of an adaptive immunity cascade. ${ }^{[79,80]}$ Preliminary clues to this theory are:

1- Dependent to the viral load, circulating Ang II level has been reported to be higher in infected patients with SARS-CoV2 than in non-infected healthy people, [81]

2- ACE2 is tissue protective for the lungs in acid- or sepsis-induced ARDS in mice, ${ }^{[82]}$

3- Mechanical-stress induced ARDS as in ventilator-induced lung injury (VILI) is correlated to activation of ACE-dependent Nox1(NADPH-oxidase1)-MK(midkine)Notch2 pathway and promoted by ACE/Ang-II/AT1R axis. ${ }^{[83,84]}$

Although contribution of RAS to evolving ARDS was previously suggested in SARS, ${ }^{[47]}$ it seems that many latent aspects of the involving pathways and the role they may play have recently been elucidated. A recent animal study in Karolinska University showed that Ang II infusion in healthy swines resulted in pathological changes identical to what happens in the lungs in COVID-19. ${ }^{[85]}$

There are three orders of RAS in our body: systemic-hormonal, tissue-local (with paracrine and autocrine effects) and cellular-subcellular (with intracrine effects). , $[23,86,87]$ Invasion of SARS-CoV2 in huge number downregulates ACE2 in the host cells outrageously so that the balance in tissue-local and cellular-subcellular RAS disrupts hyper-acutely. Accordingly, the host cells lose their ability to adapt to or defeat against the consequences of sudden rise in the content of Ang II with adequate negative feedback responses by ACE2. It is worth mentioning that in healthy people, intra-cellular Ang II content in some tissues may reach up to 1000 times higher than that of plasma. ${ }^{[88]}$ In ACE2 deficiency, Ang II is not hydrolized to angiotensin[1-7] (with its cytoprotective effect) or to other less active metabolites. Consequently, hyperacute excessive content of Ang II exerts rather untoward chaotic pathological effects in an intracrine (intracellular) and autocrine (cell to the same cell) manner and even through spilling over extracellularly, in a paracrine (cell to different neighboring endothelial, macrophages, monocytes, vascular smooth muscle cells or fibroblasts) and endocrine (cell to circulation) fashion. On the other hand, some studies demonstrated that Ang II itself downregulates ACE2 expression through internalization, lysosomal degradation and AT1R-mediated ROS activated ERK/p38 MAPK pathway which promotes TACE/ADAM17 activity as well. [89], [90, 91]

Ang II increases reactive oxygen species (ROS) through AT1R-dependent induction of NADPH oxidase (Nox), mostly Nox2 and Nox4. ${ }^{[92]}$, [93] [94] Even though ROS as a signaling molecule contributes to cell homeostasis, its overproduction may lead to cell damage. ${ }^{[95]}$ Intriguingly, ROS upregulates the production of Ang II in a positive feedback response. In deficiency of ACE2, this amplifies the production of ROS dramatically. ${ }^{[96,97]}$ ROS causes DNA damage and mitochondrial dysfunction. ${ }^{[98,99]}$ It has been reported in animal studies that Ang II through AT1R reduces mitochondrial number as well as pro-survival genes 
(Nampt and sirtuin3). ${ }^{[100]}$ ROS, in turn, through opening mitochondrial K-ATP channels and disturbing mitochondrial membrane potential, upregulates mitochondrial ROS (mtROS) production in a positive feedback response. ${ }^{[101]} \mathrm{mtROS}$ functions as a triggering signaling molecule for production of pro-inflammatory cytokines. ${ }^{[102]}$ It was reported in influenza that regulated amount of mtROS induces interferon $\mathrm{Y}$ (IFNY) to restrain infection. ${ }^{[103]}$ But when mtROS rises up excessively, striking upregulation of proinflammatory cytokines must be expected. mtROS was shown to activate NLRP3 which induces IL-1 and IL-18 production. ${ }^{[104]}$ Furthermore, ROS activates inflammatory responses by inducing redox-sensitive transcriptional factors like NF-kB and activator protein 1 (AP1). ${ }^{[86], ~[105] ~}$

Intriguingly, protein $S$ in binding with ACE2 induces ADAM-17/TACE as a sheddase to separate the ectodomain subunit of this peptidase. Shedding of ACE2 is associated with the production of TNF- $\alpha$ which was argued to be the initiating cause of inflammation of the lung in SARS. ${ }^{[44,106]}$ Over-expression of TNF- $\alpha$ in the process of hyper-acute shedding of ACE2 by ADAM17 through synergism with Ang II may aggravate inflammatory milieu by inducing oxidative stress via NF-kB and p38 MAPK dependent pathways. ${ }^{[91,107,108]}$ Furthermore, activation of AT1R by Angll induces expression of TNF$\alpha$ (presented already in the scene), IL-1 $\beta, I L-6, I L-8$, MCP-1 and even IL-10 through a NF$\mathrm{kB}$ and activating protein 1 (AP-1) transcriptional factors. ${ }^{[109,110]}$

Ang II through AT1R was also reported to amplify oxidative stress by distorting iron homeostasis and increasing labile ferrous iron as well as expression of ferritin in endothelial cells. ${ }^{[111,112]}$ Even though ferritin may show an antioxidative effect, it has been described in mice that ferritin may act as a local cytokine and activate NF-kB through MAPK-mediated pathway. This response results in a rise of inducible NO synthase for about 100 -fold and IL-1 $\beta$ and RANTES for 50 -fold with a small increase in intercellular adhesion molecule (ICAM). Ferritin may suppress adaptive immune response, as well. ${ }^{[113]}$

Aggravating to its pro-inflammatory effects, Ang II signaling through AT1R increases vascular permeability in the lung by releasing of prostaglandins and vascular endothelial growth factor (VEGF). ${ }^{[114]}$ Disruption of endothelial-epithelial (blood-air) barrier in alveoli and increase in permeability of endothelium rises the fluid in the alveolar sacs that should be cleared out by epithelial $\mathrm{Na}$ channels (ENaCs). In rats, endogenous activation of AT1R by Ang II downregulates ENaC expression and disturbs pouring out the extra fluid. [115]

Of the cytokines induced by Ang II, IL-6 plays a more special role in immunopathological effect of Ang II. IL-6 induces JAK2/STAT1/3 signaling pathway which promotes many genes contributing to the production of signaling molecules like cytokines, adaptors, receptors and protein kinases. ${ }^{[114],[116]}$ In this context, IL-6 is involved in regulation of differentiation of monocytes into macrophages, upregulation of B-cell IgG production, downregulation of dendritic cell maturation by activation of the STAT3 signaling pathway and the promotion of the Th2 response by inhibiting Th1 polarization. ${ }^{[14]}$ 
Ang II promotes production and release of IL-6 and IL-8 from human cultured adipocytes by NF-kB-mediated pathway to which AT1R rather than AT2R contributes. It is demonstrated that in the obese IL-6 plasma level is closely correlated to body mass index (BMI). ${ }^{[117]}$ Ang II-induced over expression of IL-6 in adipose tissue might be the reason why obesity is a risk factor in severity of COVID19. ${ }^{[118]}$

IL-6 has been found to increase platelet and immune cell aggregation through a T-cell dependent mechanism by Ang II. [119] In addition, an in vitro study showed Ang II to upregulate plasminogen activator inhibitors (PAls) as well as tissue plasminogen activator (TPA) mRNA in vascular smooth muscle (VSM) cell culture both directly and indirectly through PDGF. In this manner the increase in PAls not only neutralizes the effect of TPA and increases thrombophilia but the remains of TPA by resorbing extra-cellular matrix provides the possibility for the immune cell migration to the inflammation site. ${ }^{[120]}$ Moreover, apart from the ability of Ang II to stimulate platelet aggregation in normotensive and hypertensive subjects, this vasoactive substance could change of the shape of platelets derived from healthy volunteers in in vitro studies; this effect of Angll on increasing of mean platelet volume (MPV) which is considered the first step of platelet activation does not respond to aspirin. ${ }^{[121,122]}$ Molecular and cellular endocrinology findings in previous studies show that the theory of Ang II-mediated immunopathology in COVID 19 could explain the thrombophilia observed in this disease. ${ }^{[123]}$

Plasma IL-6 level has been correlated to the severity of COVID19. ${ }^{[124]}$ This cytokine, in association with IL-1 and TNF- $\alpha$, is the major inducer of CRP production in the liver ${ }^{[125,}$ 126] In addition, Ang II induces CRP expression in hepatocytes in a time- and dosedependent manner through activation of AT1R and resulting from ROS-MAPK-(NF-kb) pathway independent of IL-1 $\beta$ and IL-6. ${ }^{[127]}$ Reciprocally, CRP increases expression of AT1R in vascular smooth muscle cells with subsequent upregulation of ROS. ${ }^{[128]}$

Ang II through AT1R-PKA-proteosome pathway as well as activation of STAT1 and NF$\mathrm{kB}$ promotes differentiation of Th0 to Th1. It has been demonstrated that in shifting from Th0 to Th1 or Th2, Ang II upregulates the production of IFNy (10-fold), IL-2 (18-fold), IL4 (3.5-fold) and IL-10 (1.5-fold). In addition, Ang II increases Tbox transcription factor mRNA (Tbet, marker for Th1) and GATA3 mRNA (marker for Th2) by 38 and 1.6-fold, respectively. Amazingly, losartan, an AT1R blocker, has been shown to inhibit Th1 differentiation without having any effect on that of Th2. ${ }^{[129]}$ It is noteworthy that Th1 is differentiated to Th17 in the presence of IL- 6 and TGF- $\beta$. ${ }^{[114],[129]}$ Th17 induces synthesis of IL-17, IL-21, IL-22 and TNF- $\alpha$. TNF- $\alpha$, itself in the presence of IL- 6 and IL-1 $\beta$ may promote differentiation of T cells to Th17. ${ }^{[129,130]}$ High level of IL-17 in patients with ARDS suggests its contribution to this syndrome. This cytokine in a model of influenza and LPS induced acute lung injury has been associated with neutrophil recruitment and increased alveolar layer permeability. ${ }^{[131]}$

Local RAS has been found in DCs, T and NK cells with a complete enzymatic repertoire enabling them to synthesize and metabolize Ang II and even AT1R and AT2R. ${ }^{[132-135]}$ It has also been described that these cells not only respond to Ang II but they have tendency 
to migrate to this peptide. Thenceforth, Ang II may orchestrate recruitment of leukocytes to the site of inflammation. Besides, Ang II induces synthesis of CCL5/RANTES chemokine in T cells and NK cells. It shows that Ang II may direct chemotaxis of cells possessing CCR1, CCR3 and CCR5 and even regulate proliferation of $\mathrm{T}$ cells via CCR5. ${ }^{[136]}$

Activation of AT1R by Ang II has been correlated with apoptosis in pneumocytes. ${ }^{[137]}$ Consequent alveolar and bronchial cell death contributes to the pathogenesis of SARSCoV2. But cell loss would ultimately restrain the distribution of the virus. To make scene ready for the virus to continue its replication a reasonable percentage of the cells should survive. It merits mentioning that IL-6 in synergism with IL-17 (secreted by Th17) induces expression of pro-survival proteins $\mathrm{Bcl}-2$ and $\mathrm{Bcl}-\mathrm{xl}$ which inhibit cell destruction by CD8+ cytotoxic $T$ cells and prevent apoptosis of pneumocytes. ${ }^{[138]}$ Moreover, IL-22, a member of IL-10 anti-inflammatory cytokine family, secreted by Th17 also prevents apoptosis of pulmonary endothelial cells and ameliorates ARDS through inducing JAK2/STAT3

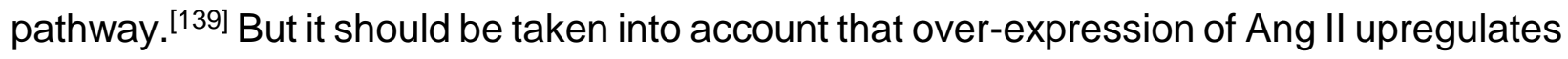
pro-inflammatory cytokines [IFNY (10-fold) and IL-2 (18 fold)] much more than antiinflammatory cytokines [IL-4 (3.5-fold) and IL-10 (1.5-fold)]. ${ }^{[129]}$ It seems that in this milieu of highly complicated set of pro-inflammatory cytokines some anti-inflammatory molecules prevent apoptosis of the respiratory epithelial and may even help giant cells produced in COVID 19 to survive.

Ang II induced upregulation of matrix metalloproteinases (MMPs) in vascular smooth muscle cells results in destruction of the pulmonary tissue including the interstitial and basal collagen-elastin structures. AT1R-mediated extra-cellular signal-regulated kinase 1/2 (ERK1/2) and AT1R-ROS mediated NF-kB and AP-1 pathways lead to increase in expression and tissue content of MMPs. These proteinases regulate remodeling and turnover of extra-cellular matrix (ECM) and promote smooth muscle and endothelial cell proliferation and migration resulting in vascular wall fibrosis which eventually may end up in pulmonary hypertension if their presence lasts. ${ }^{[140-143]}$ It has been reported in an animal study that ACE2 deficiency results in activation MMP and STAT3 pathway which may promote lung injuries. ${ }^{[144]}$

In oxidative stress macrophages exhibit more AT1R. Activating AT1R impairs efferocytosis (clearance of apoptotic cells) and interferes with resolution of the inflammatory cascade. ${ }^{[145,146]}$ MerTK is a tyrosine kinase which shifts DCs from proinflammatory to anti-inflammatory status. ${ }^{[147]}$ Ang II reduces MerTK content of the cell membrane through shedding of MerTk in a AT1R/ROS/p38MAPK/ADAM17 mediated pathway. ${ }^{[148]}$ Consequently, Ang II impairs switching M1 (with more pro-inflammatory abilities) to M2 (with more anti-inflammatory and tissue repairing properties). Continuation of pro-inflammatory status result in activation of MMPs and inducing of ECM remodeling processes. Failure of Tregs to show up due to the predominance of IL-6 promoting Th17 may prevent effective efferocytosis. In this milieu, pro-fibrotic IL-13 and TGF- $\beta$ may lead to lung fibrosis. Ang II through AT1R induces fibrotic changes in the lungs with direct and 
indirect effects. It has been shown in transgenic mice that Ang II stimulates lung fibroblasts/myofibroblast proliferation and synthesis of ECM. It induces production of TGF- $\beta$ and connective tissue growth factor (CTGF). ${ }^{[149,150]}$ Ang II in synergism with TGF$\beta$ may promote fibrosis in many organs including the lungs. Moreover, Angll-induced oxidative stress may promote pulmonary fibrosis through downregulation of sirtuin3. [151, 152] On the other hand, angiotensin [1-7], the product of ACE2, has been described as an antifibrotic molecule in cardiomyocytes through stimulation of Sirtuin3-dependent deacetylation of Foxo3a. ${ }^{[34,153]}$ In a recent study deletion of sirtuin3 accentuated Angllmediated tissue stiffness and induced arterial pericyte to fibroblast transition through activation of AT1R. ${ }^{[154]}$

\section{Losartan in cytokine storm and ARDS in COVID-19}

According to all above-mentioned studies it is rationally expected that losartan as an AT1R blocker might attenuate ARDS and cytokine storm in COVID-19. Ang II was previously considered as a factor that plays a role in ARDS and losartan is effective in ameliorating this pathology. ${ }^{[155-157]}$ However, there are also some studies considering inconsistency of ARB effects in attenuating inflammatory reactions in vascular bed. ${ }^{[158,}$ 159] But these studies were assessing ARB effects on the probable metabolic-based medium- to long-term atherosclerotic vascular inflammatory reactions but in the milieu where hydrolyzing of Angll by ACE2 was still possible. It merits mentioning that in some other studies anti-oxidant effect of losartan and its ability to reduce vascular inflammatory markers are discernible. ${ }^{[160,161]}$ Anyhow, it should be notified that what is encountered in COVID19 might hypothetically be a sudden dramatic downregulation of ACE2 due to invasion of huge number of virions and subsequent striking upregulation of Ang II followed by huge increase in AT1R content by Ang II in a positive feedback manner. Losartan was effective in many studies in suppressing pro-inflammatory effects of cytokines due to its immunomodulatory properties or even in preventing lung fibrosis. ${ }^{[143,147,162-164]}$

Moreover, losartan inhibits Ang II dependent change of shape (not responding to aspirin) and aggregation of platelets or independent to Ang II through its antagonistic effect against thromboxane A2 more than irbesartan, telmisartan, valsartan, candesartan and olmesartan .[122, 165, 166]

Early in pandemic of COVID-19 it was suggested that ARBs might increase viral load upregulation of ACE2 by these drugs seen in previous studies. ${ }^{[167-169]}$ It should be notified that overexpression of ACE2 after administration of ARBs needed 25-28 days to appear. Furthermore, according to an animal study this might be the upregulation of AT1R and PKC $\sigma$ which increases the severity of COVID-19 in hypertensive patients with chronic ARBs intake. ${ }^{[170]}$ On the other hand, entry of the virus to the cells needs that TMPRSS2 increases simultaneous to upregulation of ACE2 otherwise overexpression of ACE2 alone is capable of entrapping and decreasing the infectivity of the virus. ${ }^{[171-173]}$ Accordingly, scientific societies in Europe and the USA recommended that ARBs be continued in 
patients with hypertension or heart diseases. ${ }^{[174]}$ Conclusively, BRACE CORONA trial recently showed safety of ARBs in COVID-19. [175] [176]

As a preliminary clinical evidence of curing effect of losartan in COVID-19, two slices of lung CT-scan of a patient with COVID-19 whom was admitted to ICU, Bazarganan Hospital, Tehran, Iran, are shown. It is obviously seen that ground glass opacities cleared out significantly within four days after administration of losartan (6.25 mg twice a day). The patient's dyspnea subsided drastically after three doses of losartan.(Figure 7)

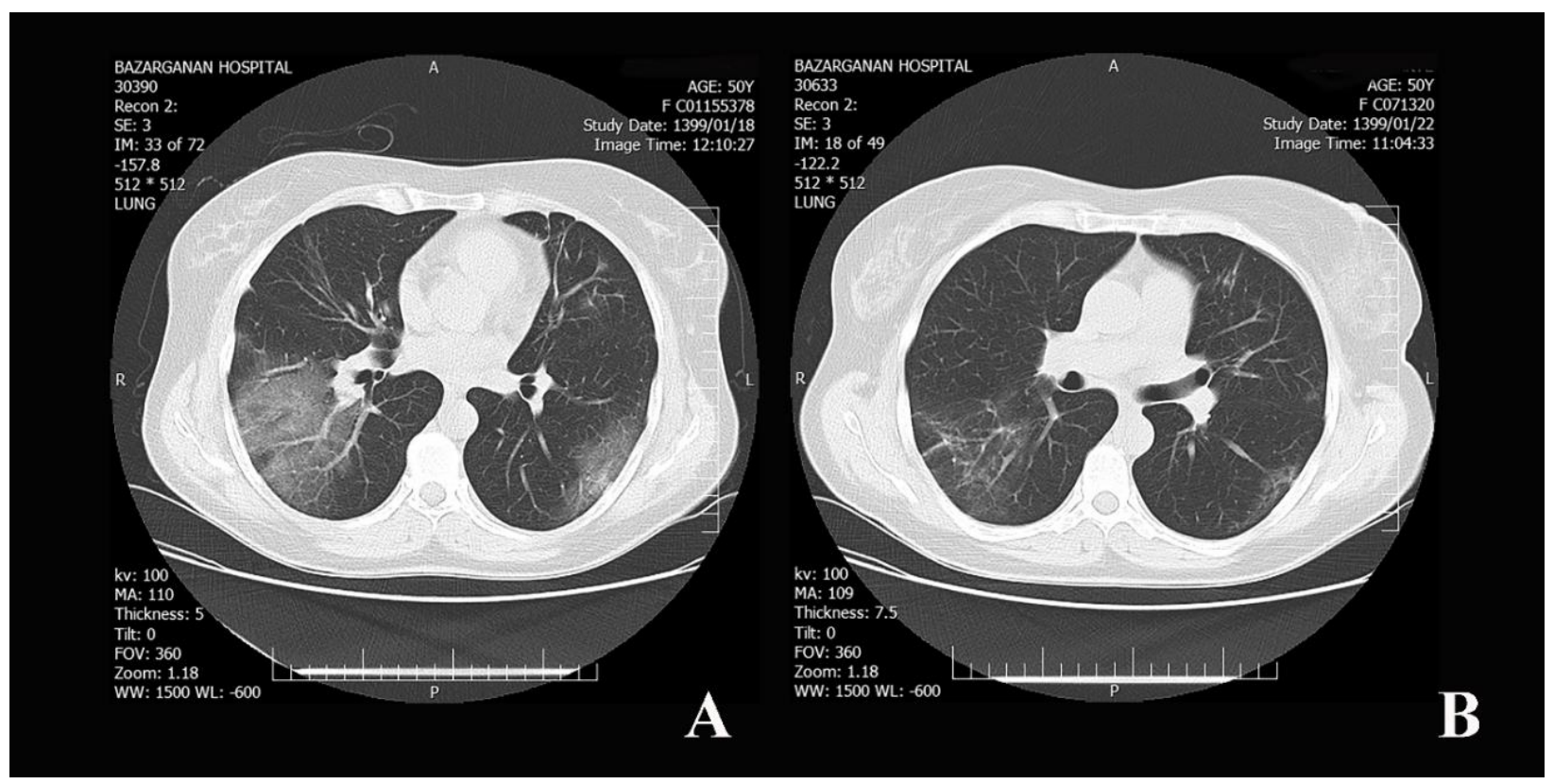

Figure 7. Spiral lung CT-scan of a 50 years old patient with COVID19; A: Apr 6, 2020; B: Apr 10, 2020

\section{Imatinib in cytokine storm and ARDS in COVID-19}

As the patients with cytokine storm may experience hypotension due to superimposed infection in the extreme severity of the disease and losartan administration may reduce the blood pressure it seems to be rational to recommend an immunomodulator in association with losartan to stop the cytokine storm more effectively. There is conflicting evidence that systemic corticosteroids may be hazardous to patients with COVID 19. [177] As Abelson tyrosine-protein kinase 2 (Abl 2) is needed in replication of SARS-CoV and MERS-CoV., tyrosine kinase inhibitors has been introduced in the treatment of SARS, MERS. In this family of viruses, imatinib hinders the initial phases of the virion replication by inhibiting fusion of the virion at the endosomal membrane. ${ }^{[178,179]}$ Imatinib, with immunomodulatory effects, has also been suggested to have subsiding effects specially against ARDS and the vascular leak seen in this syndrome. ${ }^{[131,180-182]}$ There is also a report that low dose imatinib was effective in reducing pulmonary blood pressure in dogs. ${ }^{[183]}$ In another report inhaled imatinib was also used as a drug to subside pulmonary hypertension. ${ }^{[184]}$ 
Amazingly, Imatinib was found to have an inhibitory effect against Ang II impact on vascular smooth muscle cells in dissection of the aorta in mice. ${ }^{[185]}$ Furthermore, expression of MHC class I and II, production of co-stimulatory molecules and secretion of cytokines and chemokines in monocyte-derived dendritic cells decrease in the presence of imatinib. This tyrosine kinase inhibitor subsides phosphatidylinositol 3kinase/Akt pathways and downregulates exhibition of NF-kB in the nucleus. ${ }^{[186]}$ Cultured human monocytes are morphologically and functionally suppressed in the presence on imatinib which reduces the ability of these cells to synthesize IL-6 and TNF- $\alpha$ and to respond efficiently to M-CSF and GM-CSF stimulation. ${ }^{[187]}$ In an in vitro study, imatinib could inhibit expression of TNF- $\alpha$, IL-6, IFNy and IL-17 in cultured splenocyte of mice with arthritis in a dose dependent manner. ${ }^{[188]}$ In monocytes and macrophages, TNF- $\alpha$ production was reduced by imatinib while IL-10 expression did not change. ${ }^{[189]}$ Imatinib in mice with hyper-reactive airway disease could subside peri-bronchial eosinophil accumulation and decrease secretion of IL- 4 and IL-13 by Th2 as well as CCL2, CCL5 and CCL6 chemokines. ${ }^{[190]}$ In vitro, imatinib could impair immunosuppressive ability and expression of FoxP+ in Tregs along with the subsiding of STAT3 and STAT5 pathways without affecting IL-10 and TGF- $\beta$ in these cells. ${ }^{[191]}$ Besides, in low dosage, imatinib may elicit a physiologic innate immune response to infection called "emergency response" in which myelopoiesis, but not lymphopoiesis, is potentiated. ${ }^{[192]}$ Imatinib inhibits not only S protein-dependent virus-cell fusion but also prevents syncytia and giant cell formation in the lungs. ${ }^{[193]}$

\section{In Silico Study}

We conducted an In Silico modeling study to investigate the probable inhibitory or modulatory effect of losartan and imatinib in some critical points of the replication cycle of SARS-CoV2. It was elucidated that both losartan and imatinib could bind to ACE2 with higher affinity relative to the "Pre-inh"' (reference ligand, table 2). It does not mean that these drugs could inhibit the catalytic property of this carboxypeptidase as it has not been reported, yet.

As a novel finding, we have demonstrated that losartan and imatinib could distort the binding site of SARS-CoV2 RBD to ACE2. According to our study there are seven points on the $\alpha$-helix arm of ACE2 molecule located between glycine 24 and lysine 353 (fig 2A and 2D, table 3) where SARS-CoV2 RBD (acceptor) and ACE2 (donor) may establish hydrogen $(\mathrm{H})$ bonds. The distance between the acceptor-donor residues at these points

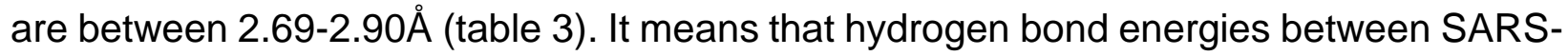
CoV2 RBD and ACE2 based on the distance of acceptor and donor residues, without considering the hydrophobic bonds, are of moderate magnitude and mostly electrostatic. ${ }^{[194]}$ The high affinity of losartan and imatinib to bind with ACE2 which increases even more after 100ns of exposure of these two drugs to ACE2 in MD simulation, show that the bonds are stable enough. According to table 3, a-helix of ACE2 was shown to be relocated for at least $1.80 \AA$ in the presence of losartan and imatinib in 
100 ns MD simulation. But in some binding residues the change after relocation was more significant (more than $3.00 \AA$ ) which may weaken $\mathrm{H}$-bonds at these points:

1- for losartan in four out of seven binding points,

2- for imatinib in six out of seven binding points.

According to this modeling it is expected that the affinity of the virus to its receptor might decrease in the presence of these two drugs. In addition, our study showed that losartan among other available ARBs in the market has the highest affinity to ACE2 (table 5). It is implicated that other ARBs might not be efficacious in docking to ACE2 to make stable similar conformational structural change in the receptor of the virus.

Furthermore, we could find that both losartan and imatinib could pose in Mpro, PLpro and MAPK molecules in the position of their "Pre-inh"' with higher affinity. These docking energies in our model are important because they determine how these ligands may probably affect the behavior of the proteases.

Of all the proposed ligands, imatinib could pose favorably in furin structure with higher affinity relative to its "Pre-inh". Considering high expression of furin in the lungs and importance of S'2 cleavage ${ }^{[17]}$ in the entry of SARS-CoV2 to host cell, if the inhibitory effect of imatinib against furin is approved in in vivo studies it can be regarded as an inhibitor that hinders entry of SARS-CoV2 to the target cells, as well. Imatinib and losartan due to their effective docking to PLpro and Mpro with higher affinity relative to "Pre-inh" might be successful in preventing those proteases from letting the virus evade innate immunity or start replication. Although in the case of PLpro the docking energy for losartan were higher (lower affinity) than that of "Pre-inh", the magnitude of energies (<-9.21) was low enough to consider losartan as a probable inhibitor of PLpro. RMSD diagram for $100 \mathrm{~ns}$ MD simulation of losartan-PLpro complex showed that a sudden change of about $0.25 \mathrm{~nm}(2.5 \AA)$ in the mean position of PLpro molecules occurred in about 60ns that continued till the end of MD simulation for $100 \mathrm{~ns}$ with a fall in affinity or in other words rise in the docking energy [from -9.21 (Kcal/mole) before MD simulation to -7.83 (Kcal/mole) post-hoc] (Figure 9). This may indicate that losartan affects the conformational shape and probably the function of PLpro at the expense of losing its affinity to the protein to some degree. ${ }^{[195,196]}$ Perhaps longer dynamic study is needed to explain the real behavior of PLpro in the presence of losartan.

Bradykinin induces allergic inflammatory responses with activation of airway fibroblast/myofibroblasts through MAPK pathway. ${ }^{[197,}{ }^{198]}$ In many of the pathophysiological destructive pathways in COVID 19 the signature of MAPK is evident. Surprisingly, in this modeling In Silico study, losartan and imatinib showed to have significant tendency to bind with p38 MAPK. This might indicate that these drug ligands may have inhibitory effect against p38MAPK and its downstream pathways and may inhibit untoward bradykinin-dependent or responses, as well. In our In Silico study, imatinib also showed its tendency to bind to Ang II (figure 10). It should be investigated if imatinib could change the function of Ang II. 


\section{Conclusion}

Through binding the scattered rings of previously found evidence, our reviewing the literature showed that hyper-acute activation of AT1R by a sudden rise of intracellular Ang II due to downregulation of ACE2 after showering of SARS-CoV2 to the lungs explains precisely the pathophysiology of cytokine storm and ARDS in COVID-19. It is implied that administration of losartan, an AT1R blocker, and imatinib, an immunomodulator and as an adjunctive to losartan might be promising in the attenuating ARDS in COVID-19.

Our In Silico study showed that losartan and imatinib may decrease the affinity of the virus to ACE2 and might have inhibitory effects against furin, PLpro and MAPK to be validated in in-vitro, subclinial and clinical studies in the future

According to the findings in this study and the preliminary clinical evidences, we suggest low dose systemic losartan and inhaled aerosolized low dose imatinib be studied in a subclinical setting in treating ARDS in COVID 19. In this manner while losartan by blocking AT1R antagonizes Ang II effects, aerosolized imatinib may modulate the local immunological responses. Furthermore, this combination may reduce the probability of binding of the virus to ACE2 more effectively.

\section{References:}

1. Chan, J.F.-W., et al., Genomic characterization of the 2019 novel human-pathogenic coronavirus isolated from a patient with atypical pneumonia after visiting Wuhan. Emerging microbes \& infections, 2020. 9(1): p. 221-236.

2. Viruses., C.S.G.o.t.I.C.o.T.o., The species Severe acute respiratory syndrome-related coronavirus: classifying 2019-nCoV and naming it SARS-CoV-2. Version 2. . Nature Microbiology, 2020. 5(4): p. 8.

3. Gorbalenya, A.E., et al., The species Severe acute respiratory syndrome-related coronavirus: classifying 2019-nCoV and naming it SARS-CoV-2. Nature Microbiology, 2020. 5(4): p. 536-544.

4. Cui, J., F. Li, and Z.-L. Shi, Origin and evolution of pathogenic coronaviruses. Nature reviews Microbiology, 2019. 17(3): p. 181-192.

5. Snijder, E., E. Decroly, and J. Ziebuhr, The nonstructural proteins directing coronavirus RNA synthesis and processing, in Advances in virus research. 2016, Elsevier. p. 59-126.

6. Mielech, A.M., et al., Nidovirus papain-like proteases: multifunctional enzymes with protease, deubiquitinating and delSGylating activities. Virus research, 2014. 194: p. 184190.

7. Fehr, A.R. and S. Perlman, Coronaviruses: an overview of their replication and pathogenesis, in Coronaviruses. 2015, Springer. p. 1-23.

8. Lei, J., Y. Kusov, and R. Hilgenfeld, Nsp3 of coronaviruses: Structures and functions of a large multi-domain protein. Antiviral research, 2018. 149: p. 58-74.

9. Nejat, R. and A.S. Sadr, SARS Virus Papain-like Protease: A Mysterious Weapon. Journal of Biostatistics and Epidemiology, 2019. 5(4): p. 288-295. 
10. Shin, D., et al., Papain-like protease regulates SARS-CoV-2 viral spread and innate immunity. Nature, 2020: p. 1-10.

11. Blanco-Melo, D., et al., SARS-CoV-2 launches a unique transcriptional signature from in vitro, ex vivo, and in vivo systems. BioRxiv, 2020.

12. Yoshikawa, T., et al., Dynamic innate immune responses of human bronchial epithelial cells to severe acute respiratory syndrome-associated coronavirus infection. PloS one, 2010. 5(1).

13. Li, F., Structure, function, and evolution of coronavirus spike proteins. Annual review of virology, 2016. 3: p. 237-261.

14. Ou, X., et al., Characterization of spike glycoprotein of SARS-CoV-2 on virus entry and its immune cross-reactivity with SARS-CoV. Nature communications, 2020. 11(1): p. 1-12.

15. White, J.M., et al., Structures and mechanisms of viral membrane fusion proteins: multiple variations on a common theme. Critical reviews in biochemistry and molecular biology, 2008. 43(3): p. 189-219.

16. Zhou, P., et al., A pneumonia outbreak associated with a new coronavirus of probable bat origin. nature, 2020. 579(7798): p. 270-273.

17. Wrapp, D., et al., Cryo-EM structure of the 2019-nCoV spike in the prefusion conformation. Science, 2020. 367(6483): p. 1260-1263.

18. Jaimes, J.A., J.K. Millet, and G.R. Whittaker, Proteolytic cleavage of the SARS-CoV-2 spike protein and the role of the novel S1/S2 site. Iscience, 2020: p. 101212.

19. Coutard, B., et al., The spike glycoprotein of the new coronavirus 2019-nCoV contains a furin-like cleavage site absent in CoV of the same clade. Antiviral research, 2020. 176: p. 104742.

20. $\mathrm{Li}, \mathrm{W}$., et al., Angiotensin-converting enzyme 2 is a functional receptor for the SARS coronavirus. Nature, 2003. 426(6965): p. 450-454.

21. Wan, Y., et al., Receptor recognition by the novel coronavirus from Wuhan: an analysis based on decade-long structural studies of SARS coronavirus. Journal of virology, 2020. 94(7).

22. $\mathrm{Li}, \mathrm{W}$., et al., Receptor and viral determinants of SARS-coronavirus adaptation to human ACE2. The EMBO journal, 2005. 24(8): p. 1634-1643.

23. Marshall, R.P., The pulmonary renin-angiotensin system. Current pharmaceutical design, 2003. 9(9): p. 715-722.

24. Parodi-Rullan, R., et al., Direct renin inhibition exerts an anti-hypertrophic effect associated with improved mitochondrial function in post-infarction heart failure in diabetic rats. Cellular Physiology and Biochemistry, 2012. 29(5-6): p. 841-850.

25. Goossens, G., et al., Angiotensin II: a hormone that affects lipid metabolism in adipose tissue. International journal of obesity, 2007. 31(2): p. 382-384.

26. Satou, R., H. Penrose, and L.G. Navar, Inflammation as a regulator of the renin-angiotensin system and blood pressure. Current hypertension reports, 2018. 20(12): p. 100.

27. Donoghue, M., et al., A novel angiotensin-converting enzyme-related carboxypeptidase (ACE2) converts angiotensin I to angiotensin 1-9. Circulation research, 2000. 87(5): p. e1e9.

28. Reddy Gaddam, R., S. Chambers, and M. Bhatia, ACE and ACE2 in inflammation: a tale of two enzymes. Inflammation \& Allergy-Drug Targets (Formerly Current Drug TargetsInflammation \& Allergy), 2014. 13(4): p. 224-234. 
29. Riviere, G., et al., Angiotensin-converting enzyme 2 (ACE2) and ACE activities display tissue-specific sensitivity to undernutrition-programmed hypertension in the adult rat. Hypertension, 2005. 46(5): p. 1169-1174.

30. Arendse, L.B., et al., Novel Therapeutic Approaches Targeting the Renin-Angiotensin System and Associated Peptides in Hypertension and Heart Failure. Pharmacological reviews, 2019. 71(4): p. 539-570.

31. Dilauro, M. and K.D. Burns, Angiotensin-(1-7) and its effects in the kidney. The Scientific World Journal, 2009. 9: p. 522-535.

32. Bader, M., ACE2, angiotensin-(1-7), and Mas: the other side of the coin. Pflügers ArchivEuropean Journal of Physiology, 2013. 465(1): p. 79-85.

33. Watanabe, T., T.A. Barker, and B.C. Berk, Angiotensin I/ and the endothelium: diverse signals and effects. Hypertension, 2005. 45(2): p. 163-169.

34. Uhal, B.D., et al., Angiotensin signalling in pulmonary fibrosis. The international journal of biochemistry \& cell biology, 2012. 44(3): p. 465-468.

35. Reddy, R., et al., Circulating angiotensin peptides levels in Acute Respiratory Distress Syndrome correlate with clinical outcomes: A pilot study. PloS one, 2019. 14(3).

36. Lee, S.H., et al., Angiotensin II-induced oxidative stress in human endothelial cells: modification of cellular molecules through lipid peroxidation. Chemical research in toxicology, 2019. 32(7): p. 1412-1422.

37. Benigni, A., P. Cassis, and G. Remuzzi, Angiotensin II revisited: new roles in inflammation, immunology and aging. EMBO molecular medicine, 2010. 2(7): p. 247-257.

38. Manabe, S., et al., Effects of angiotensin II receptor blockade with valsartan on proinflammatory cytokines in patients with essential hypertension. Journal of cardiovascular pharmacology, 2005. 46(6): p. 735-739.

39. Dandona, P., et al., Angiotensin II and inflammation: the effect of angiotensin-converting enzyme inhibition and angiotensin II receptor blockade. Journal of human hypertension, 2007. 21(1): p. 20-27.

40. Santos, R., et al., Angiotensin-converting enzyme 2, angiotensin-(1-7) and Mas: new players of the renin-angiotensin system. J endocrinol, 2013. 216(2): p. R1-R17.

41. Imai, Y., K. Kuba, and J.M. Penninger, The discovery of angiotensin-converting enzyme 2 and its role in acute lung injury in mice. Experimental physiology, 2008. 93(5): p. 543-548.

42. Dominici, F.P., et al., Modulation of the action of insulin by angiotensin-(1-7). Clinical science, 2014. 126(9): p. 613-630.

43. Jia, H., Pulmonary angiotensin-converting enzyme 2 (ACE2) and inflammatory lung disease. Shock, 2016. 46(3): p. 239-248.

44. Glowacka, I., et al., Differential downregulation of ACE2 by the spike proteins of severe acute respiratory syndrome coronavirus and human coronavirus NL63. Journal of virology, 2010. 84(2): p. 1198-1205.

45. Deshotels, M.R., et al., Angiotensin II mediates angiotensin converting enzyme type 2 internalization and degradation through an angiotensin ii type $i$ receptor-dependent mechanism. Hypertension, 2014. 64(6): p. 1368-1375.

46. Kelly, D.J., et al., Platelet-derived growth factor receptor transactivation mediates the trophic effects of angiotensin II in vivo. Hypertension, 2004. 44(2): p. 195-202.

47. Imai, Y., K. Kuba, and J.M. Penninger, The renin-angiotensin system in acute respiratory distress syndrome. Drug Discovery Today: Disease Mechanisms, 2006. 3(2): p. 225-229. 
48. Gao, B.-B., et al., Angiotensin II stimulates phosphorylation of an ectodomain-truncated platelet-derived growth factor receptor- $B$ and its binding to class IA PI3K in vascular smooth muscle cells. Biochemical Journal, 2006. 397(2): p. 337-344.

49. Berman, H.M., et al., The protein data bank. Nucleic acids research, 2000. 28(1): p. 235242.

50. Lan, J., et al., Structure of the SARS-CoV-2 spike receptor-binding domain bound to the ACE2 receptor. Nature, 2020: p. 1-6.

51. Towler, P., et al., ACE2 X-ray structures reveal a large hinge-bending motion important for inhibitor binding and catalysis. Journal of Biological Chemistry, 2004. 279(17): p. 1799618007.

52. Jin, Z., et al., Structure of Mpro from COVID-19 virus and discovery of its inhibitors. bioRxiv. 2020.

53. Wang, Z., et al., Structural basis of inhibitor selectivity in MAP kinases. Structure, 1998. 6(9): p. 1117-1128.

54. Van Lam van, T., et al., Design, synthesis, and characterization of macrocyclic inhibitors of the proprotein convertase furin. ChemMedChem, 2019. 14(6): p. 673-685.

55. Ghosh, A.K., et al., Severe acute respiratory syndrome coronavirus papain-like novel protease inhibitors: design, synthesis, protein- ligand $X$-ray structure and biological evaluation. Journal of medicinal chemistry, 2010. 53(13): p. 4968-4979.

56. Wingler, L.M., et al., Angiotensin and biased analogs induce structurally distinct active conformations within a GPCR. Science, 2020. 367(6480): p. 888-892.

57. Pettersen, E.F., et al., UCSF Chimera-a visualization system for exploratory research and analysis. Journal of computational chemistry, 2004. 25(13): p. 1605-1612.

58. DeLano, W.L., The PyMOL molecular graphics system. http://www. pymol. org, 2002.

59. Guex, N. and M.C. Peitsch, SWISS-MODEL and the Swiss-Pdb Viewer: an environment for comparative protein modeling. electrophoresis, 1997. 18(15): p. 2714-2723.

60. Kim, S., et al., PubChem 2019 update: improved access to chemical data. Nucleic acids research, 2019. 47(D1): p. D1102-D1109.

61. Wishart, D.S., et al., DrugBank 5.0: a major update to the DrugBank database for 2018. Nucleic acids research, 2018. 46(D1): p. D1074-D1082.

62. Nagy, Á., Density functional. Theory and application to atoms and molecules. Physics Reports, 1998. 298(1): p. 1-79.

63. Becke, A.D., Density-functional exchange-energy approximation with correct asymptotic behavior. Physical review A, 1988. 38(6): p. 3098.

64. McKean, D., et al., Infrared and Raman spectra of 1, 1-dibromodisilanes and STO-3G* calculations. Spectrochimica Acta Part A: Molecular and Biomolecular Spectroscopy, 1996. 52(2): p. 199-205.

65. Frisch, M., et al., Gaussian 03, Revision C. 02. Wallingford, CT: Gaussian. Inc.[Google Scholar], 2004.

66. O'Boyle, N.M., et al., Open Babel: An open chemical toolbox. Journal of cheminformatics, 2011. 3(1): p. 33.

67. Morris, G.M., et al., AutoDock4 and AutoDockTools4: Automated docking with selective receptor flexibility. Journal of computational chemistry, 2009. 30(16): p. 2785-2791.

68. Berendsen, H.J., D. van der Spoel, and R. van Drunen, GROMACS: a message-passing parallel molecular dynamics implementation. Computer physics communications, 1995. 91(1-3): p. 43-56. 
69. van Gunsteren, W.F., X. Daura, and A.E. Mark, Encyclopedia of Computational Chemistry, chapter GROMOS force field. 1998, New York: John Wiley and Sons.

70. Kanehisa, M. and S. Goto, KEGG: kyoto encyclopedia of genes and genomes. Nucleic acids research, 2000. 28(1): p. 27-30.

71. Dolatkhah, Z., et al., Synthesis, molecular docking, molecular dynamics studies, and biological evaluation of 4 h-chromone-1, 2, 3, 4-tetrahydropyrimidine-5-carboxylate derivatives as potential antileukemic agents. Journal of chemical information and modeling, 2017. 57(6): p. 1246-1257.

72. Turner, P., XMGRACE, Version 5.1. 19. Cent. Coast. land-margin Res. oregon Grad. Inst. Sci. Technol. Beaverton, Ore, USA, 2005.

73. Wallace, A.C., R.A. Laskowski, and J.M. Thornton, LIGPLOT: a program to generate schematic diagrams of protein-ligand interactions. Protein engineering, design and selection, 1995. 8(2): p. 127-134.

74. Stierand, K. and M. Rarey, PoseView--molecular interaction patterns at a glance. Journal of cheminformatics, 2010. 2(1): p. 1-1.

75. Pan, Y., et al., Viral load of SARS-CoV-2 in clinical samples. The Lancet infectious diseases, 2020. 20(4): p. 411-412.

76. Hamming, I., et al., Tissue distribution of ACE2 protein, the functional receptor for SARS coronavirus. A first step in understanding SARS pathogenesis. The Journal of Pathology: A Journal of the Pathological Society of Great Britain and Ireland, 2004. 203(2): p. 631-637.

77. Sims, A.C., et al., Severe acute respiratory syndrome coronavirus infection of human ciliated airway epithelia: role of ciliated cells in viral spread in the conducting airways of the lungs. Journal of virology, 2005. 79(24): p. 15511-15524.

78. Kuba, K., et al., A crucial role of angiotensin converting enzyme 2 (ACE2) in SARS coronavirus-induced lung injury. Nature medicine, 2005. 11(8): p. 875-879.

79. Tisoncik, J.R., et al., Into the eye of the cytokine storm. Microbiology and Molecular Biology Reviews, 2012. 76(1): p. 16-32.

80. Tufet, M., T cells calm the storm. Nature Reviews Immunology, 2007. 7(11): p. 834-835.

81. Liu, Y., et al., Clinical and biochemical indexes from 2019-nCoV infected patients linked to viral loads and lung injury. Science China Life Sciences, 2020. 63(3): p. 364-374.

82. Imai, Y., et al., Angiotensin-converting enzyme 2 protects from severe acute lung failure. Nature, 2005. 436(7047): p. 112-116.

83. Zhang, R., et al., Mechanical stress and the induction of lung fibrosis via the midkine signaling pathway. American journal of respiratory and critical care medicine, 2015. 192(3): p. 315-323.

84. Wang, D., et al., Renin-angiotensin-system, a potential pharmacological candidate, in acute respiratory distress syndrome during mechanical ventilation. Pulmonary pharmacology \& therapeutics, 2019. 58: p. 101833.

85. Rysz, S., et al., COVID-19 pathophysiology may be driven by a loss of inhibition of the Renin-Angiotensin-Aldosterone System. 2020.

86. Re, R.N., Role of intracellular angiotensin II. American Journal of Physiology-Heart and Circulatory Physiology, 2018. 314(4): p. H766-H771.

87. Abadir, P.M., et al., Identification and characterization of a functional mitochondrial angiotensin system. Proceedings of the National Academy of Sciences, 2011. 108(36): p. 14849-14854. 
88. Fazeli, G., et al., Angiotensin II induces DNA damage via AT1 receptor and NADPH oxidase isoform Nox4. Mutagenesis, 2012. 27(6): p. 673-681.

89. Palau, V., M. Riera, and M.J. Soler, ADAM17 inhibition may exert a protective effect on COVID-19. Nephrology Dialysis Transplantation, 2020.

90. Koka, V., et al., Angiotensin II up-regulates angiotensin l-converting enzyme (ACE), but down-regulates ACE2 via the AT1-ERK/p38 MAP kinase pathway. The American journal of pathology, 2008. 172(5): p. 1174-1183.

91. Patel, V.B., et al., Angiotensin II induced proteolytic cleavage of myocardial ACE2 is mediated by TACE/ADAM-17: a positive feedback mechanism in the RAS. Journal of molecular and cellular cardiology, 2014. 66: p. 167-176.

92. Garrido, A.M. and K.K. Griendling, NADPH oxidases and angiotensin I/ receptor signaling. Molecular and cellular endocrinology, 2009. 302(2): p. 148-158.

93. Bernard, K., et al., NADPH oxidases in lung health and disease. Antioxidants \& redox signaling, 2014. 20(17): p. 2838-2853.

94. Rosenfeld, C.R., et al., Meconium increases type 1 angiotensin II receptor expression and alveolar cell death. Pediatric research, 2008. 63(3): p. 251-256.

95. Ray, P.D., B.-W. Huang, and Y. Tsuji, Reactive oxygen species (ROS) homeostasis and redox regulation in cellular signaling. Cellular signalling, 2012. 24(5): p. 981-990.

96. Chen, Q., et al., Reactive oxygen species: key regulators in vascular health and diseases. British journal of pharmacology, 2018. 175(8): p. 1279-1292.

97. Zhang, H. and A. Baker, Recombinant human ACE2: acing out angiotensin II in ARDS therapy. 2017, Springer.

98. Cooke, M.S., et al., Oxidative DNA damage: mechanisms, mutation, and disease. The FASEB Journal, 2003. 17(10): p. 1195-1214.

99. Cadet, J. and K.J. Davies, Oxidative DNA damage \& repair: an introduction. Free Radical Biology and Medicine, 2017. 107: p. 2-12.

100. Benigni, A., et al., Disruption of the Ang II type 1 receptor promotes longevity in mice. The Journal of clinical investigation, 2009. 119(3): p. 524-530.

101. Vajapey, R., et al., The impact of age-related dysregulation of the angiotensin system on mitochondrial redox balance. Frontiers in physiology, 2014. 5: p. 439.

102. Naik, E. and V.M. Dixit, Mitochondrial reactive oxygen species drive proinflammatory cytokine production. Journal of Experimental Medicine, 2011. 208(3): p. 417-420.

103. Kim, H.J., et al., Reactive oxygen species induce antiviral innate immune response through IFN- $\lambda$ regulation in human nasal epithelial cells. American journal of respiratory cell and molecular biology, 2013. 49(5): p. 855-865.

104. Shi, Q., et al., Mitochondrial ROS activate interleukin-16 expression in allergic rhinitis. Oncology letters, 2018. 16(3): p. 3193-3200.

105. Bernstein, K.E., et al., Angiotensin-converting enzyme in innate and adaptive immunity. Nature Reviews Nephrology, 2018. 14(5): p. 325.

106. Haga, S., et al., Modulation of TNF- $\alpha$-converting enzyme by the spike protein of SARS-CoV and ACE2 induces TNF- $\alpha$ production and facilitates viral entry. Proceedings of the National Academy of Sciences, 2008. 105(22): p. 7809-7814.

107. Takahashi, M., et al., Angiotensin II and tumor necrosis factor- $\alpha$ synergistically promote monocyte chemoattractant protein-1 expression: roles of $N F-K B, p 38$, and reactive oxygen species. American Journal of Physiology-Heart and Circulatory Physiology, 2008. 294(6): p. $\mathrm{H} 2879-\mathrm{H} 2888$. 
108. Mayr, M., et al., TNF/Ang-Il synergy is obligate for fibroinflammatory pathology, but not for changes in cardiorenal function. Physiological reports, 2016. 4(8): p. e12765.

109. Sauter, N.S., et al., Angiotensin II induces interleukin-16-mediated islet inflammation and b-cell dysfunction independently of vasoconstrictive effects. Diabetes, 2015. 64(4): p. 1273-1283.

110. Guo, F., et al., Role of angiotensin II type 1 receptor in angiotensin II-induced cytokine production in macrophages. Journal of interferon \& cytokine research, 2011. 31(4): p. 351-361.

111. Ishizaka, N., et al., Iron chelation suppresses ferritin upregulation and attenuates vascular dysfunction in the aorta of angiotensin II-infused rats. Arteriosclerosis, thrombosis, and vascular biology, 2005. 25(11): p. 2282-2288.

112. Tajima, S., et al., Effect of angiotensin II on iron-transporting protein expression and subsequent intracellular labile iron concentration in human glomerular endothelial cells. Hypertension Research, 2010. 33(7): p. 713-721.

113. Kernan, K.F. and J.A. Carcillo, Hyperferritinemia and inflammation. International immunology, 2017. 29(9): p. 401-409.

114. Velazquez-Salinas, L., et al., The role of interleukin 6 during viral infections. Frontiers in microbiology, 2019. 10: p. 1057.

115. Deng, J., et al., The effect of endogenous angiotensin II on alveolar fluid clearance in rats with acute lung injury. Canadian respiratory journal, 2012. 19(5): p. 311-318.

116. Weidanz, J., et al., AT1R blockade reduces IFN-p production in lymphocytes in vivo and in vitro. Kidney international, 2005. 67(6): p. 2134-2142.

117. Skurk, T., V. van Harmelen, and H. Hauner, Angiotensin II stimulates the release of interleukin-6 and interleukin-8 from cultured human adipocytes by activation of NF-KB. Arteriosclerosis, thrombosis, and vascular biology, 2004. 24(7): p. 1199-1203.

118. Lighter, J., et al., Obesity in patients younger than 60 years is a risk factor for Covid-19 hospital admission. Clin Infect Dis, 2020. 9(10.1093).

119. Senchenkova, E.Y., et al., Novel Role of T Cells and IL-6 (Interleukin-6) in Angiotensin IIInduced Microvascular Dysfunction. Hypertension, 2019. 73(4): p. 829-838.

120. Van Leeuwen, R., et al., Angiotensin II increases plasminogen activator inhibitor type 1 and tissue-type plasminogen activator messenger RNA in cultured rat aortic smooth muscle cells. Circulation, 1994. 90(1): p. 362-368.

121. Touyz, R.M. and E.L. Schiffrin, Effects of angiotensin II and endothelin-1 on platelet aggregation and cytosolic $\mathrm{pH}$ and free $\mathrm{Ca} 2+$ concentrations in essential hypertension. Hypertension, 1993. 22(6): p. 853-862.

122. Jagroop, I. and D. Mikhailidis, Angiotensin II can induce and potentiate shape change in human platelets: effect of losartan. Journal of human hypertension, 2000. 14(9): p. 581585.

123. Klok, F.A., et al., Confirmation of the high cumulative incidence of thrombotic complications in critically ill ICU patients with COVID-19: An updated analysis. Thrombosis research, 2020.

124. Herold, T., et al., Level of IL-6 predicts respiratory failure in hospitalized symptomatic COVID-19 patients. medRxiv 2020. Google Scholar, 2020.

125. Eklund, C.M., Proinflammatory cytokines in CRP baseline regulation. Advances in clinical chemistry, 2009. 48: p. 111-136. 
126. Bermudez, E.A., et al., Interrelationships among circulating interleukin-6, C-reactive protein, and traditional cardiovascular risk factors in women. Arteriosclerosis, thrombosis, and vascular biology, 2002. 22(10): p. 1668-1673.

127. Zhao, J., et al., Angiotensin II induces C-reactive protein expression via AT1-ROS-MAPK-NF$K B$ signal pathway in hepatocytes. Cellular Physiology and Biochemistry, 2013. 32(3): p. 569-580.

128. Wang, C.-H., et al., C-reactive protein upregulates angiotensin type 1 receptors in vascular smooth muscle. Circulation, 2003. 107(13): p. 1783-1790.

129. Qin, X.-Y., et al., Angiotensin II regulates Th1 T cell differentiation through angiotensin II type 1 receptor-PKA-mediated activation of proteasome. Cellular Physiology and Biochemistry, 2018. 45(4): p. 1366-1376.

130. Zheng, Y., et al., TNF $\alpha$ promotes Th17 cell differentiation through IL-6 and IL-16 produced by monocytes in rheumatoid arthritis. Journal of immunology research, 2014. 2014.

131. Rizzo, A.N., et al., Targeting Abl kinases to regulate vascular leak during sepsis and acute respiratory distress syndrome. Arteriosclerosis, thrombosis, and vascular biology, 2015. 35(5): p. 1071-1079.

132. Sun, P., et al., Expression of Renin-Angiotensin System on Dendritic Cells of Patients with Coronary Artery Disease. Inflammation, 2009. 32(6): p. 347.

133. Lapteva, N., et al., Expression of renin-angiotensin system genes in immature and mature dendritic cells identified using human cDNA microarray. Biochemical and biophysical research communications, 2001. 285(4): p. 1059-1065.

134. Meng, Y., et al., Angiotensin II regulates dendritic cells through activation of NF-KB/p65, ERK1/2 and STAT1 pathways. Cellular Physiology and Biochemistry, 2017. 42(4): p. 15501558.

135. Hoch, N.E., et al., Regulation of T-cell function by endogenously produced angiotensin II. American Journal of Physiology-Regulatory, Integrative and Comparative Physiology, 2009.

136. Jurewicz, M., et al., Human T and natural killer cells possess a functional renin-angiotensin system: further mechanisms of angiotensin II-induced inflammation. Journal of the American Society of Nephrology, 2007. 18(4): p. 1093-1102.

137. Lukkarinen, H.P., et al., Angiotensin II receptor inhibition prevents pneumocyte apoptosis in surfactant-depleted rat lungs. Pediatric pulmonology, 2005. 39(4): p. 349-358.

138. Hou, W., et al., Interleukin-6 (IL-6) and IL-17 synergistically promote viral persistence by inhibiting cellular apoptosis and cytotoxic T cell function. Journal of virology, 2014. 88(15): p. 8479-8489.

139. Ren, W., et al., JAK2/STAT3 pathway was associated with the protective effects of IL-22 on aortic dissection with acute lung injury. Disease markers, 2017. 2017.

140. Browatzki, M., et al., Angiotensin II stimulates matrix metalloproteinase secretion in human vascular smooth muscle cells via nuclear factor- $k B$ and activator protein 1 in a redox-sensitive manner. Journal of vascular research, 2005. 42(5): p. 415-423.

141. Wang, C., et al., Angiotensin II increases matrix metalloproteinase 2 expression in human aortic smooth muscle cells via AT1R and ERK1/2. Experimental Biology and Medicine, 2015. 240(12): p. 1564-1571.

142. Wang, X., et al., Effects of angiotensin II intervention on MMP-2, MMP-9, TIMP-1, and collagen expression in rats with pulmonary hypertension. Genet Mol Res, 2015. 14(1): p. 1707-1717. 
143. Guo, Y.-S., et al., Impact of losartan and angiotensin II on the expression of matrix metalloproteinase-9 and tissue inhibitor of metalloproteinase-1 in rat vascular smooth muscle cells. Molecular medicine reports, 2015. 11(3): p. 1587-1594.

144. Hung, Y.-H., et al., Alternative roles of STAT3 and MAPK signaling pathways in the MMPs activation and progression of lung injury induced by cigarette smoke exposure in ACE2 knockout mice. International journal of biological sciences, 2016. 12(4): p. 454.

145. Yamamoto, S., et al., Macrophage polarization by angiotensin II-type 1 receptor aggravates renal injury-acceleration of atherosclerosis. Arteriosclerosis, thrombosis, and vascular biology, 2011. 31(12): p. 2856-2864.

146. Keidar, S., et al., Oxidative stress increases the expression of the angiotensin-Il receptor type 1 in mouse peritoneal macrophages. Journal of the Renin-Angiotensin-Aldosterone System, 2002. 3(1): p. 24-30.

147. Anwar, A., et al., Mer tyrosine kinase (MerTK) promotes macrophage survival following exposure to oxidative stress. Journal of leukocyte biology, 2009. 86(1): p. 73-79.

148. Zhang, Y., et al., Angiotensin II deteriorates advanced atherosclerosis by promoting MerTK cleavage and impairing efferocytosis through the AT1R/ROS/p38 MAPK/ADAM17 pathway. American Journal of Physiology-Cell Physiology, 2019. 317(4): p. C776-C787.

149. Wang, J., et al., Chronic activation of the renin-angiotensin system induces lung fibrosis. Scientific reports, 2015. 5: p. 15561.

150. Proto, J.D., et al., Regulatory $T$ cells promote macrophage efferocytosis during inflammation resolution. Immunity, 2018. 49(4): p. 666-677. e6.

151. Murphy, A.M., A.L. Wong, and M. Bezuhly, Modulation of angiotensin II signaling in the prevention of fibrosis. Fibrogenesis \& tissue repair, 2015. 8(1): p. 7.

152. Sosulski, M.L., et al., Sirtuin 3 deregulation promotes pulmonary fibrosis. The Journals of Gerontology: Series A, 2017. 72(5): p. 595-602.

153. Guo, L., et al., Angiotensin-(1-7) attenuates angiotensin II-induced cardiac hypertrophy via a Sirt3-dependent mechanism. American Journal of Physiology-Heart and Circulatory Physiology, 2017. 312(5): p. H980-H991.

154. Chen, J.X. and H. Zeng, Deficiency of Sirtuin 3 Accentuates Angiotensin II-Induced Arterial/Myocardial Stiffness and Hypertension. The FASEB Journal, 2019. 33(S1): p. 819.4-819.4.

155. Khan, A., et al., A pilot clinical trial of recombinant human angiotensin-converting enzyme 2 in acute respiratory distress syndrome. Critical Care, 2017. 21(1): p. 234.

156. Ruthman, C.A. and E. Festic, Emerging therapies for the prevention of acute respiratory distress syndrome. Therapeutic advances in respiratory disease, 2015. 9(4): p. 173-187.

157. Kim, J., et al., Effect of Renin-Angiotensin System Blockage in Patients with Acute Respiratory Distress Syndrome: A Retrospective Case Control Study. Korean journal of critical care medicine, 2017. 32(2): p. 154.

158. Del Fiorentino Alessandra, S.C., et al., The effect of angiotensin receptor blockers on Creactive protein and other circulating inflammatory indices in man. Vascular Health and Risk Management, 2009. 5: p. 233.

159. Chang, L.-T., et al., Impact of simvastatin and losartan on antiinflammatory effect: in vitro study. Journal of cardiovascular pharmacology, 2007. 49(1): p. 20-26.

160. Rajagopalan, S., et al., Effect of losartan in aging-related endothelial impairment. The American journal of cardiology, 2002. 89(5): p. 562-566. 
161. Graninger, M., et al., Angiotensin receptor blockade decreases markers of vascular inflammation. Journal of cardiovascular pharmacology, 2004. 44(3): p. 335-339.

162. Nahmod, K.A., et al., Control of dendritic cell differentiation by angiotensin II. The FASEB Journal, 2003. 17(3): p. 491-493.

163. Mitra, A.K., L. Gao, and I.H. Zucker, Angiotensin II-induced upregulation of AT1 receptor expression: sequential activation of NF-KB and Elk-1 in neurons. American Journal of Physiology-Cell Physiology, 2010. 299(3): p. C561-C569.

164. Wang, F., et al., Losartan inhibits LPS+ATP-induced IL-1beta secretion from mouse primary macrophages by suppressing NALP3 inflammasome. Die Pharmazie-An International Journal of Pharmaceutical Sciences, 2014. 69(9): p. 680-684.

165. Guerra-Cuesta, J.I., et al., Effect of losartan on human platelet activation. Journal of hypertension, 1999. 17(3): p. 447-452.

166. Yamada, K., T. Hirayama, and Y. Hasegawa, Antiplatelet effect of losartan and telmisartan in patients with ischemic stroke. Journal of Stroke and Cerebrovascular Diseases, 2007. 16(5): p. 225-231.

167. Ferrario, C.M., et al., Effect of angiotensin-converting enzyme inhibition and angiotensin II receptor blockers on cardiac angiotensin-converting enzyme 2. Circulation, 2005. 111(20): p. 2605-2610.

168. Soler, M.J., et al., Localization of ACE2 in the renal vasculature: amplification by angiotensin II type 1 receptor blockade using telmisartan. American Journal of PhysiologyRenal Physiology, 2009. 296(2): p. F398-F405.

169. Guo, J., et al., Coronavirus disease 2019 (covid-19) and cardiovascular disease: a viewpoint on the potential influence of angiotensin-converting enzyme inhibitors/angiotensin receptor blockers on onset and severity of severe acute respiratory syndrome coronavirus 2 infection. Journal of the American Heart Association, 2020. 9(7): p. e016219.

170. Song, M.A., C. Dasgupta, and L. Zhang, Chronic losartan treatment up-regulates AT1R and increases the heart vulnerability to acute onset of ischemia and reperfusion injury in male rats. PloS one, 2015. 10(7).

171. Sanchis-Gomar, F., et al. Angiotensin-Converting Enzyme 2 and antihypertensives (angiotensin receptor blockers and angiotensin-converting enzyme inhibitors) in Coronavirus Disease 2019. in Mayo Clinic Proceedings. 2020. Elsevier.

172. Perico, L., A. Benigni, and G. Remuzzi, Should COVID-19 concern nephrologists? Why and to what extent? The emerging impasse of angiotensin blockade. Nephron, 2020. 144(5): p. 213-221.

173. Vaduganathan, M., et al., Renin-angiotensin-aldosterone system inhibitors in patients with Covid-19. New England Journal of Medicine, 2020. 382(17): p. 1653-1659.

174. South, A.M., et al., Controversies of renin-angiotensin system inhibition during the COVID19 pandemic. Nature Reviews Nephrology, 2020: p. 1-3.

175. Lopes, R.D., et al., Continuing versus suspending angiotensin-converting enzyme inhibitors and angiotensin receptor blockers: Impact on adverse outcomes in hospitalized patients with severe acute respiratory syndrome coronavirus 2 (SARS-CoV-2)--The BRACE CORONA Trial. American Heart Journal, 2020. 226: p. 49-59.

176. Lopes, R.D., et al., Continuing Versus Suspending Angiotensin-Converting Enzyme Inhibitors and Angiotensin Receptor Blockers - BRACE CORONA. Journal of American College of Cardiology, 2020. 
177. Veronese, N., et al., Use of Corticosteroids in Coronavirus Disease 2019 Pneumonia: A Systematic Review of the Literature. Frontiers in Medicine, 2020. 7: p. 170.

178. Coleman, C.M., et al., Abelson kinase inhibitors are potent inhibitors of severe acute respiratory syndrome coronavirus and middle east respiratory syndrome coronavirus fusion. Journal of virology, 2016. 90(19): p. 8924-8933.

179. Ruella, M., et al., Kinase inhibitor ibrutinib to prevent cytokine-release syndrome after anti-CD19 chimeric antigen receptor $T$ cells for B-cell neoplasms. Leukemia, 2017. 31(1): p. 246-248.

180. Rizzo, A.N., et al., Imatinib attenuates inflammation and vascular leak in a clinically relevant two-hit model of acute lung injury. American Journal of Physiology-Lung Cellular and Molecular Physiology, 2015. 309(11): p. L1294-L1304.

181. Stephens, R.S., et al., The tyrosine kinase inhibitor imatinib prevents lung injury and death after intravenous LPS in mice. Physiological reports, 2015. 3(11): p. e12589.

182. Kim, I.K., et al., Effect of tyrosine kinase inhibitors, imatinib and nilotinib, in murine lipopolysaccharide-induced acute lung injury during neutropenia recovery. Critical Care, 2013. 17(3): p. R114.

183. Arita, S., N. Arita, and Y. Hikasa, Therapeutic effect of low-dose imatinib on pulmonary arterial hypertension in dogs. The Canadian Veterinary Journal, 2013. 54(3): p. 255.

184. Pitsiou, G., et al., Inhaled tyrosine kinase inhibitors for pulmonary hypertension: a possible future treatment. Drug design, development and therapy, 2014. 8: p. 1753.

185. Sun, T., et al., Imatinib inhibits angiotensin II-induced aortic dissection through the c-AbI signaling pathway. Int J Clin Exp Pathol, 2017. 10(5): p. 5316-5324.

186. Appel, S., et al., Effects of imatinib on monocyte-derived dendritic cells are mediated by inhibition of nuclear factor-KB and Akt signaling pathways. Clinical Cancer Research, 2005. 11(5): p. 1928-1940.

187. Dewar, A.L., et al., Imatinib inhibits the functional capacity of cultured human monocytes. Immunology and cell biology, 2005. 83(1): p. 48-56.

188. Berlin, A.A. and N.W. Lukacs, Treatment of cockroach allergen asthma model with imatinib attenuates airway responses. American journal of respiratory and critical care medicine, 2005. 171(1): p. 35-39.

189. Wolf, A.M., et al., The kinase inhibitor imatinib mesylate inhibits TNF- $\alpha$ production in vitro and prevents TNF-dependent acute hepatic inflammation. Proceedings of the National Academy of Sciences, 2005. 102(38): p. 13622-13627.

190. Akashi, N., et al., Comparative suppressive effects of tyrosine kinase inhibitors imatinib and nilotinib in models of autoimmune arthritis. Modern rheumatology, 2011. 21(3): p. 267-275.

191. Larmonier, N., et al., Imatinib mesylate inhibits CD4+CD25+ regulatory $T$ cell activity and enhances active immunotherapy against BCR-ABL-tumors. The Journal of Immunology, 2008. 181(10): p. 6955-6963.

192. Napier, R.J., et al., Low doses of imatinib induce myelopoiesis and enhance host antimicrobial immunity. PLoS pathogens, 2015. 11(3).

193. Sisk, J.M., M.B. Frieman, and C.E. Machamer, Coronavirus S protein-induced fusion is blocked prior to hemifusion by Abl kinase inhibitors. The Journal of general virology, 2018. 99(5): p. 619.

194. Jeffrey, G.A. and G.A. Jeffrey, An introduction to hydrogen bonding. Vol. 12. 1997: Oxford university press New York. 
195. Oferkin, I.V., et al., Evaluation of docking target functions by the comprehensive investigation of protein-ligand energy minima. Advances in bioinformatics, 2015. 2015.

196. Pantsar, T. and A. Poso, Binding affinity via docking: fact and fiction. Molecules, 2018. 23(8): p. 1899.

197. Golias, C., et al., The kinin system-bradykinin: biological effects and clinical implications. Multiple role of the kinin system-bradykinin. Hippokratia, 2007. 11(3): p. 124.

198. Sabatini, F., et al., Bradykinin-induced asthmatic fibroblast/myofibroblast activities via bradykinin $B 2$ receptor and different MAPK pathways. European journal of pharmacology, 2013. 710(1-3): p. 100-109.

\section{Supplementary}

Table S 1. The list of required FDA-Approved drugs

\begin{tabular}{|l|l|l|}
\hline Small Molecules & Pubchem CID & Drug bank \\
\hline Imatinib & 5291 & DB00619 \\
\hline Losartan & 3961 & DB00678 \\
\hline
\end{tabular}

Table S 2. Configurations of computational systems

\begin{tabular}{|c|c|c|c|}
\hline System & Operating Systems & CPU & GPU \\
\hline \multirow[t]{2}{*}{ Server 1} & \multirow[t]{2}{*}{ Linux 3.10} & $\begin{array}{ll}32 & \text { logical } \\
\text { cores } & \\
\end{array}$ & \multirow[t]{2}{*}{ CUDA } \\
\hline & & E5-2697 & \\
\hline \multirow[t]{2}{*}{ Server 2} & \multirow[t]{2}{*}{ Linux 4.15} & $\begin{array}{ll}32 & \text { logical } \\
\text { cores } & \end{array}$ & \multirow[t]{2}{*}{$\times$} \\
\hline & & E5-2650 & \\
\hline
\end{tabular}


$\mathbf{A}$

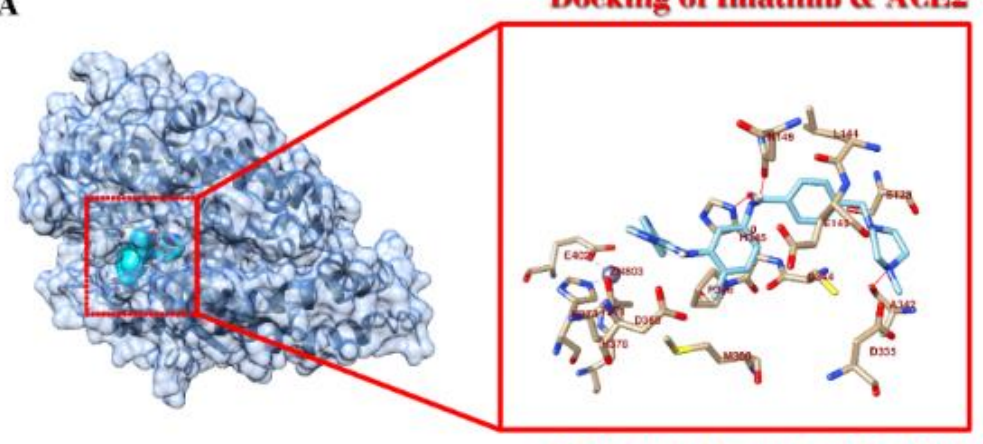

B

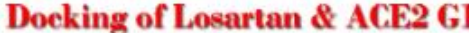

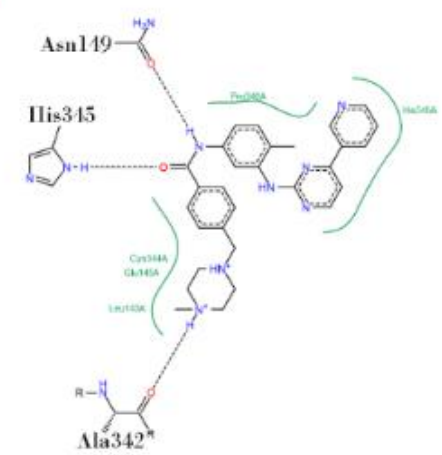

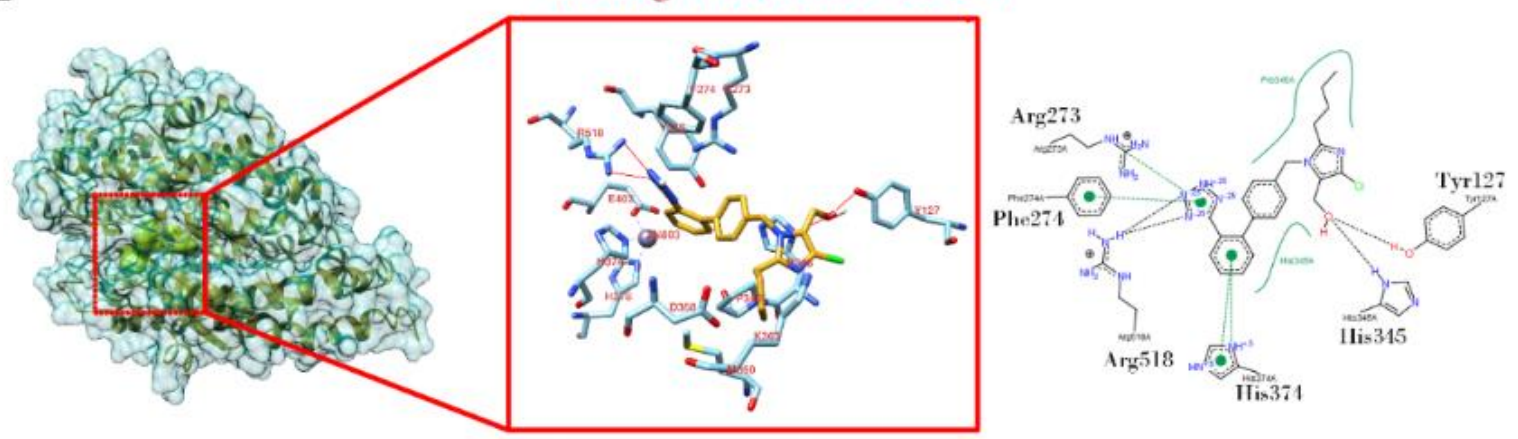

Figure S 1. Docking of Imatinib and Losartan (before MD simulation); A: binding site of imatinib with ACE2 with hydrogen bonds, Asn149, Ala342, His345; B: binding site of losartan with ACE2 including hydrogen bonds, Tyr127, Arg273, Arg374, His345 and pi-pi interactions of Phe274 and His374 

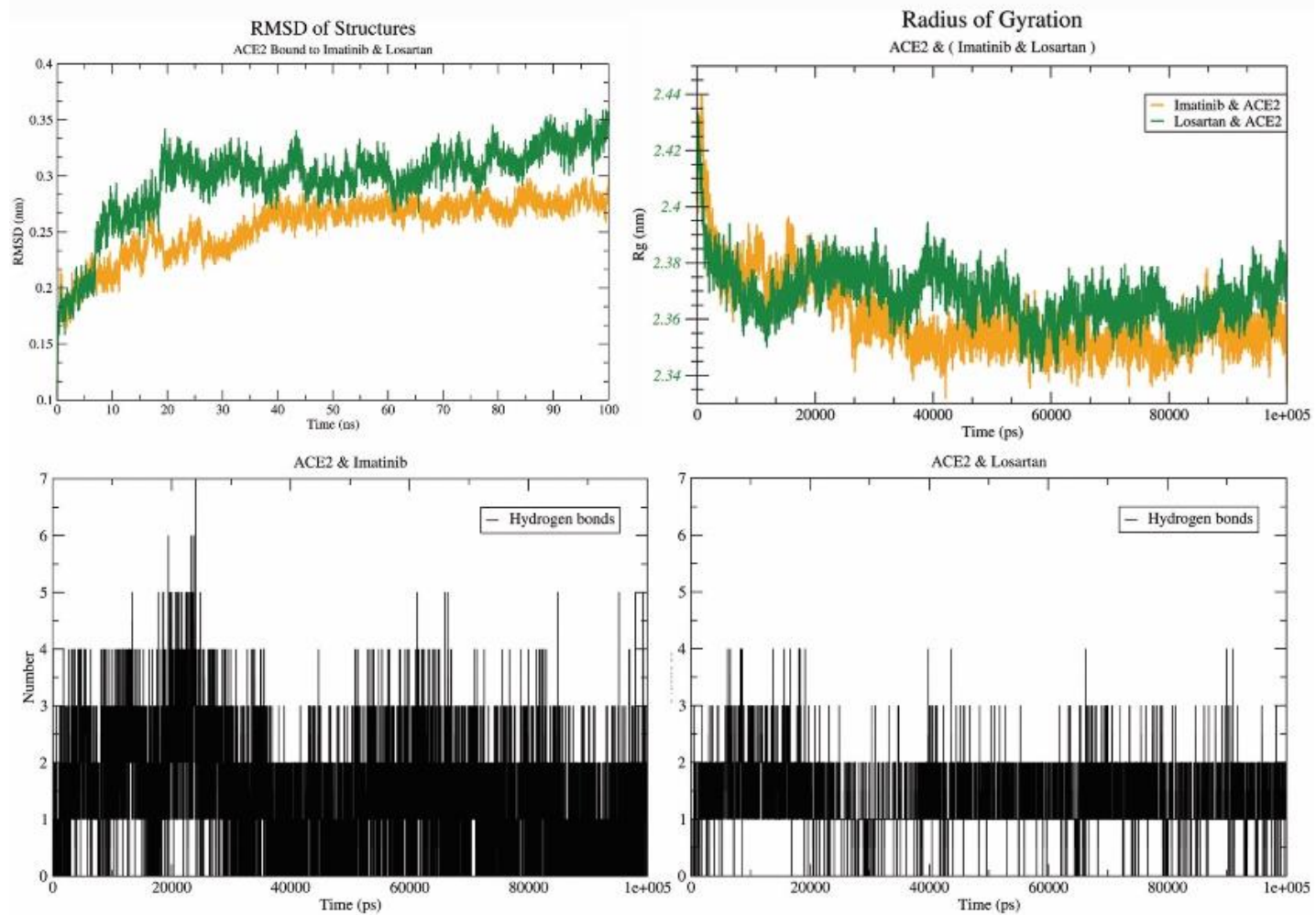

Figure S 2. RMSD, Rg and H-bonding diagrams of losartan and imatinib complexes with ACE2

Table S 3. Relocation of Carbon alpha of N-terminal helix of ACE2

\begin{tabular}{|c|c|c|c|c|c|}
\hline \multirow[b]{2}{*}{ RBD } & \multirow[b]{2}{*}{ H-Bond length (Å) } & \multirow[b]{2}{*}{ Hydrophobic } & \multirow[b]{2}{*}{ Atom $C(\alpha)$} & \multicolumn{2}{|c|}{ ACE2 Residue Relocation Magnitudes } \\
\hline & & & & Imatinib (Å) & Losartan (Å) \\
\hline Asn487(R) & 2.69 & & GLN 24. A & 6.32 & 6.06 \\
\hline \multirow[t]{2}{*}{ Lys417(R) } & 2.90 & & ASP 30.A & 3.46 & 3.24 \\
\hline & - & $\checkmark$ & HIS 34.A & 2.59 & 2.37 \\
\hline Tyr449(R) & 2.70 & & ASP 38.A & 3.69 & 2.18 \\
\hline Thr500(R) & 2.71 & & TYR 41.A & 1.98 & 1.80 \\
\hline Tyr49(R) & 2.79 & & GLN 42.A & 3.33 & 2.05 \\
\hline Asn487(R) & 2.69 & & Tyr 83.A & 7.58 & 6.50 \\
\hline Gly502 (R) & 2.78 & & Lys 353.A & 4.04 & 3.99 \\
\hline \multicolumn{3}{|c|}{ N-Terminal } & SER 19.A & 9.24 & 7.71 \\
\hline
\end{tabular}


Table S 4. . Docking Energy for binding ARBs to ACE2

\begin{tabular}{|lr}
\hline ARBs & Binding Energy \\
\hline Pratosartan & -9.97 \\
\cline { 1 - 1 } Tasosartan & -9.74 \\
\cline { 1 - 1 } Losartan & -9.6 \\
\cline { 1 - 1 } Candesartan & -9.43 \\
\hline Irbesartan & -9.25 \\
\hline Fimasartan & -9.07 \\
\hline Telmisartan & -8.82 \\
\hline Forasartan & -8.77 \\
\hline Azilsartan & -8.4 \\
\hline Saprisartan & -7.9 \\
\hline Olmesartan & -7.62 \\
\hline Valsartan & -7.39 \\
\hline Eprosartan & -6.62 \\
\hline Saralasin & -3.69 \\
\hline
\end{tabular}

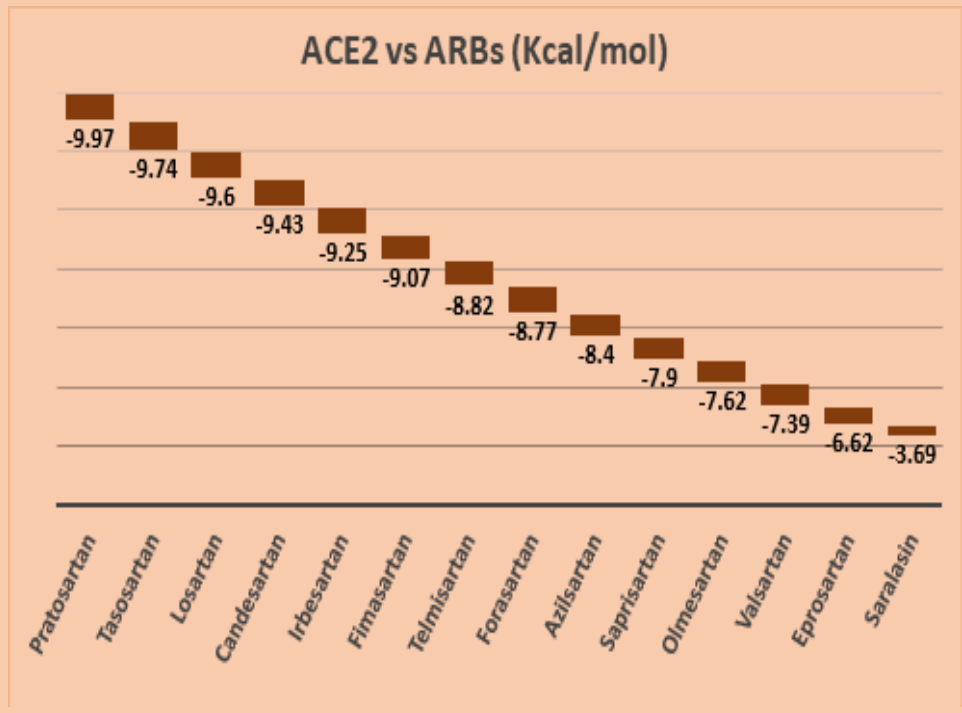

SARS-CoVI CPFGEVFNATKFPSVYAWERKKI SNCVADYSVLYNSTFFSTFKCYGVS SARS-CDV2 LICPFGEVFNATRFASVYAWNRKR I SNCVADYSVLYNSASFSTFKCYGVSP SARS-CoVl. AT. KL . NDLCF SN ...VYADSFVVKGDDVRQI APGQTGVI ADYNYKLPD SARS-CoV2 TKLND. LC.......FT NVYADSFVIRGDEVRQI APGQTGKI ADYNYKLPD SARS-CoVI D F MG C VLAWNTRN I DAT ST GNYNYKYRYLRHGKLRPFERD I SNVPFSPDG SARS-CoV2 DFTGCVI AWNSNNLDS.... Y Y Y LYR.

SARS-CoVI KPCTPPA L NCYWPLNDYGFYTTTG I GYQPYRVVVLSFE.

SARS-COV2 ........... PLQSYGFQPT. VGYQPYRVVVLSFEL L H A $\dot{P} \dot{A} T \dot{T} \dot{C} \dot{G} \dot{P} \dot{K}$

Figure $S$ 3. Sequence alignment of RBD of SARS-CoV and SARS-CoV2 for crystal structures by PDBID:3sci and PDBID: 6vsb 


\section{Angiotensin II - Imatinib Interaction}

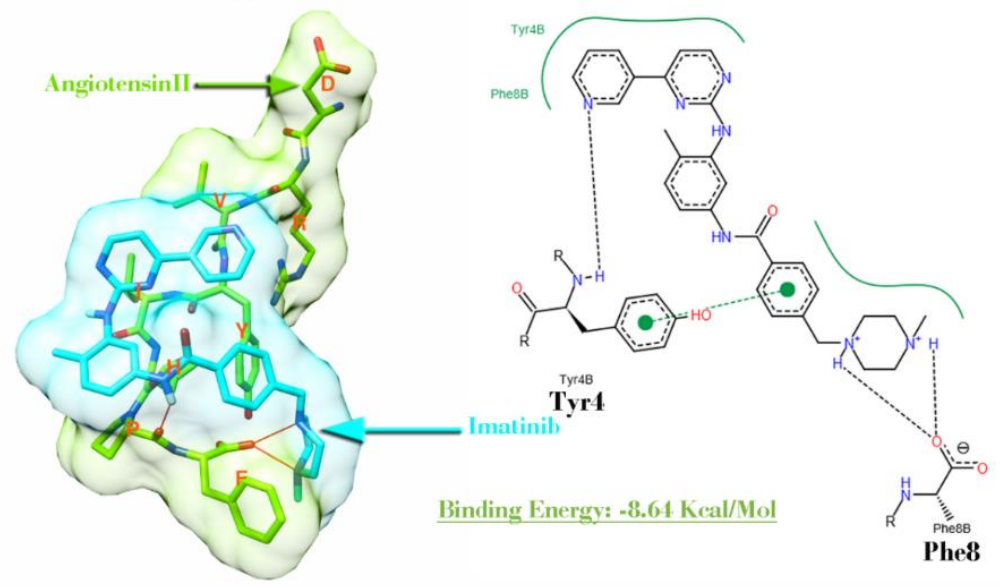

Figure S 4. . Angiotensin II-Imatinib Interaction in Surface view and poseview of interaction with two hydrogen bonds with Phe8 and pi-pi interaction with Tyr4 